\title{
Linearisation Method of DML-based Transmitters for Optical Communications Part I: Theory and Simulation Studies
}

\author{
N. Bamiedakis, D. G. Cunningham, Member, IEEE, and R.V. Penty, Senior Member, IEEE
}

\begin{abstract}
The performance of directly-modulated lasers (DMLs) is severely impaired by nonlinear behaviour when operating at high symbol rates. We propose a new linearization method for DML-based transmitters which can significantly reduce nonlinearity. This method, named the "Stretched A" (StrA) method, relies on the generation of an approximation to the ideal modulating current that generates a linear optical output waveform. In Part I of this work, the theoretical framework of the proposed method is presented and detailed simulation studies illustrate its implementation and demonstrate the benefits it offers. Although the method is applicable to any type of DML, the simulation studies presented herein focus on optical links based on vertical-cavity surface-emitting lasers (VCSELs) as these comprise the vast majority of short-reach optical links. Part II of this work presents the proof-of-principle experimental demonstration of this new linearization method and discusses its possible implementations using either analog or digital electronics.
\end{abstract}

Index Terms - directly-modulated lasers, optical links, linearisation method, optical transmitters, non linearity, vertical cavity surface emitting lasers

\section{INTRODUCTION}

$\mathrm{T}$ The ever increasing demand for data communication capacity drives the need for higher data rate optical links in both the short- and long-reach domain [1]. Although nowadays long-reach telecommunication links primarily deploy externally modulated optical sources, short-reach datacommunication links are largely based on directlymodulated lasers (DMLs) due to their low cost and ease of implementation. However, the performance of such optical links is severely impaired by the intrinsic non-linearity of DMLs when operated at high data rates such that the symbol rate is greater than the $3 \mathrm{~dB}$ bandwidth. This results in significant non-linear distortion of the output optical waveform and degradation of the link performance. The nonlinearity of the signal manifests as skew in the optical eye diagram, thickening of the optical levels, overshoot and increased jitter. To accommodate these effects, the optical

Manuscript received March xx, 2021; revised May xx, 2021; accepted June xx, 2021. Date of publication December xx 2021; date of current version November xx, 2021.

N. Bamiedakis, D.G. Cunningham and R. V. Penty are with the Centre for Photonic Systems, Electrical Engineering Division, Department of Engineering, University of Cambridge, Cambridge, CB3 0FA, UK.

(e-mail: nb301@cam.ac.uk). Digital Object Identifier xxxx power budget must be increased to allow for the associated optical power penalties making the implementation of highdata rate links particularly challenging.

Whilst linear equalization, both in the optical transmitter and the optical receiver, is now commonly used in optical links, it cannot mitigate the high frequency dynamic nonlinearity of the DML. As a result, there has been intense research investigating methods to mitigate the non-linearity and the implementation of non-linear equalization methods in such links. Such methods include asymmetric feedforward equalizers [2-8] and non-linear equalization based on Volterra series equalizers [9-12], look up tables [13-16], or machinelearning algorithms [17-20]. However, all these methods are complex, power hungry and require some sort of digital signal processing (DSP) either at the transmitter or the receiver. As a result, their applicability and potential in low-cost optical links is limited. In this work we present a novel linearization method of optical transmitters based on DMLs which can be easily implemented in practice.

The proposed method, named the "Stretched A" (StrA) method, is based on generating a modulating current waveform that approximates the ideal modulating current that produces a linear optical output waveform. Shaping the modulating current waveform has been proposed as a method to improve the high speed performance of DMLs [21], and a few different laser drivers have been developed to apply waveform shaping at the transmitter [22-24]. However the currents generated in these schemes are sub-optimal. Furthermore, it has been demonstrated that the ideal current waveform can be back-calculated from the standard laser rate equations under reasonable approximations $[15,16]$. However, the obtained expression includes terms which are hard to generate in practice. A few implementations of this method have been reported in the literature, but these rely heavily on DSP to generate the desired current modulation waveforms $[15,16,25]$. As a result, its applicability and potential use in its full form in low-cost data communication links is limited. Our method is an extension of the ideal back-calculated current method and involves the simplification of the expressions that define the ideal modulating current in two steps, the ABCD approximation and StrA methods. This allows much lower implementation complexity at the transmitter than the full back-calculation method as the terms that need to be generated are of simpler form, while enabling the generation of a sufficiently accurate current waveform that adequately corrects the non-linearity in the output optical 
waveform. It is shown herein that the approximate current modulating waveform can be generated with large enough tolerances to make this scheme viable for real-world implementation.

Although the proposed method is applicable to any type of DML, the studies presented here are focused on vertical cavity surface emitting laser (VCSEL)-based links, as these comprise the large majority of short-reach optical links [26]. Typically, such links have lengths $\leq 300 \mathrm{~m}$ and combine multimode (MM) VCSELs with multimode fiber (MMF) to achieve low cost and power efficiency [27, 28]. Single mode VCSELs and fibre (SMF) are currently also being considered for use in slightly longer reach links in the range 1 to $3 \mathrm{~km}$ in data centre environments [29-31]. On a per optical lane basis, currently deployed MM VCSEL-based links can transmit $25 \mathrm{~Gb} / \mathrm{s}$ using non-return-to -zero (NRZ) or $50 \mathrm{~Gb} / \mathrm{s}$ using four level pulse amplitude modulation (PAM-4). Various standards bodies have begun projects which aim to specify per optical lane data rates for $50 \mathrm{~Gb} / \mathrm{s}$ VCSEL-based links using NRZ modulation and $100 \mathrm{~Gb} / \mathrm{s}$ using PAM-4 [32, 33]. As a result, we focus our simulation studies on the generation of high-quality $50 \mathrm{~Gb} / \mathrm{s}$ NRZ optical signals using an $850 \mathrm{~nm}$ MM VCSEL and the proposed Stretched A method. The simulation results show that a high-signal quality optical waveform can be generated which exhibits little residual non-linearity and which is very similar to the ideal linear optical waveform. A root meansquared error (RMSE) between the generated and target optical waveform of 0.005 is achieved at $50 \mathrm{~Gb} / \mathrm{s}$. The respective RMSE value for conventional NRZ modulation is 0.111 . In addition, significant improvements in the quality of the eye diagram are demonstrated in comparison to conventional NRZ modulation with an improvement of $\sim 78 \%$ in eye height, $\sim 34 \%$ in eye width and $\sim 140 \%$ in the effective area obtained for $50 \mathrm{~Gb} / \mathrm{s}$ data transmission. The simulations also demonstrate the method's great potential for real-world implementation. Although the Stretched A method presented here can be readily extended to generate high-quality PAM-4 optical waveforms, the related analysis and experimental demonstration will be presented in a separate publication.

The remainder of the paper is structured as follows. Section II presents the basic theory and derivation of the expression for the modulating current for the Stretched A method while Section III describes and illustrates the required current waveforms using a simulation model. Section IV focuses on the implementation of the Stretched A method and its tolerances while Section V discusses some related important points. Finally, Section VI provides the conclusions.

\section{THEORY}

\section{A. ABCD method}

The laser output waveform $P(t)$ is proportional to photon density $N_{p}(t)$ in the laser cavity:

$$
P(t)=\frac{V \eta_{d} h v}{2 \Gamma \tau_{p}} \cdot N_{p}(t)=\eta \cdot N_{p}(t)
$$

where $V$ is the volume of the active region, $\eta_{d}$ is the differential quantum efficiency, $\Gamma$ is the optical confinement factor, $\tau_{p}$ is the photon lifetime, $v$ is the light frequency, $h$ is Planck's constant and $\eta$ is the resulting total conversion efficiency of the laser. As a result, for a particular target output waveform $P(t)$, the required photon density $N_{p}(t)$ can be obtained. The photon density $N_{p}(t)$ can be in turn related to the carrier density $N_{e}(t)$ in the laser cavity via the standard single mode laser rate equations:

$$
\begin{gathered}
\frac{d N_{p}(t)}{d t}=\frac{\Gamma g_{0}\left(N_{e}(t)-N_{0}\right)}{1+\varepsilon \cdot N_{p}(t)} \cdot N_{p}(t)-\frac{N_{p}(t)}{\tau_{p}}+\frac{\Gamma \beta N_{e}(t)}{\tau_{e}} \\
\frac{d N_{e}(t)}{d t}=\frac{\mathrm{I}(t)}{q V}-\frac{N_{e}(t)}{\tau_{e}}-\frac{g_{0}\left(N_{e}(t)-N_{0}\right)}{1+\varepsilon \cdot N_{p}(t)} \cdot N_{p}(t)
\end{gathered}
$$

where $q$ is the charge of an electron, $N_{0}$ is the carrier density at transparency, $g_{0}$ is the gain slope coefficient, $\varepsilon$ is the gain compression factor, $\beta$ is spontaneous emission factor and $\tau_{e}$ is the carrier lifetime. It has been shown by Karar et al. [16, 34] that by reversing the laser rate equations using a backcalculation method, the required ideal carrier density $N_{e}^{b c}(t)$ and the corresponding modulation current $I_{b c}(t)$ required to produce the desired photon density $N_{p}(t)$ and therefore, the target output optical waveform $P(t)$, can be found and accurately approximated by:

$$
\begin{gathered}
N_{e}^{b c}(t)=\frac{\frac{d N_{p}}{d t}+\frac{N_{p}(t)}{\tau_{p h}}+\frac{\Gamma \cdot g_{0} \cdot N_{0}}{1+\varepsilon N_{p}(t)} \cdot N_{p}(t)}{\frac{\Gamma \cdot g_{0}}{1+\varepsilon N_{p}(t)} \cdot N_{p}(t)+\frac{\Gamma \cdot \beta}{\tau_{e}}} \\
I_{b c}(t)=q V\left(\frac{d N_{e}^{b c}}{d t}+\frac{g_{0} N_{p}(t)}{1+\varepsilon N_{p}(t)}\left(N_{e}^{b c}(t)-N_{0}\right)+\frac{N_{e}^{b c}(t)}{\tau_{e}}\right)
\end{gathered}
$$

Assuming that the spontaneous emission rate is much smaller than that of stimulated emission $\left(\frac{\Gamma \cdot \beta}{\tau_{e}} \ll \frac{\Gamma \cdot g_{0}}{1+\varepsilon N_{p}} \cdot N_{p}\right)$, which is true for operation far from the threshold current, and substituting (4) into (5), the ideal back-calculated modulating current $I_{b c}(t)$ can be expressed as a function of the photon density $N_{p}(t)$ in the laser cavity and its first and second order derivatives: $N_{p}^{\prime}=\frac{d N_{p}(t)}{d t}, N_{p}^{\prime \prime}=\frac{d^{2} N_{p}(t)}{d t^{2}}$. It can be shown that $I_{b c}(t)$ is the sum of four components:

$$
I_{b c}(t)=I_{A}(t)+I_{B}(t)+I_{c}(t)+I_{D}
$$

These four components, from which the back-calculation method is named here the "ABCD" method, are:

(i) the constant term $I_{D}$ which is equal to the threshold current:

$$
I_{D}=\frac{q V}{\tau_{e}} \cdot\left(N_{0}+\frac{1}{\Gamma \cdot g_{0} \cdot \tau_{p}}\right)=I_{t h}
$$

(ii) the linear term $I_{C}(t)$ which is proportional to the photon density $N_{p}(t)$ :

$$
I_{C}=\frac{q V}{\Gamma \tau_{p}} \cdot N_{p}(t) \cdot\left(1+\frac{\varepsilon}{g_{0} \cdot \tau_{e}}\right)
$$

(iii) the term $I_{B}(t)$ which is practically proportional to the first order derivative $N_{p}^{\prime}$ :

$$
I_{B}(t)=\frac{q V}{\Gamma} \cdot N_{p}^{\prime} \cdot\left(1+\frac{\varepsilon}{g_{0} \tau_{p}}+\frac{1+\varepsilon N_{p}}{g_{0} \cdot \tau_{e} \cdot N_{p}}\right)
$$

(iv) the term $I_{A}(t)$ which includes the higher order derivatives and the non-linear terms: 


$$
I_{A}(t)=\frac{q V}{\Gamma g_{0}} \cdot\left(\frac{N_{p}^{\prime \prime}}{N_{p}}-\left(\frac{N_{p}^{\prime}}{N_{p}}\right)^{2}+\varepsilon N_{p}^{\prime \prime}\right)
$$

An equivalent form of eq. (6) is given in [15], approximating the ideal total current $I_{b c}(t)$ with the desired target optical waveform $P(t)$ :

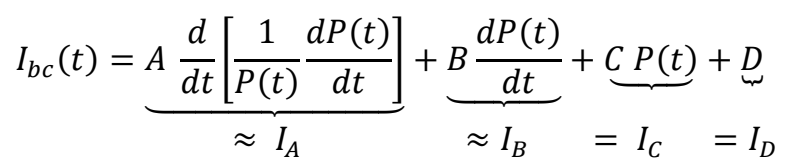

where $A, B, C$ and $D$ are constants of proportionally which can be related to the parameters which specify the rate equation for the laser.

If the target optical waveform is linear, the dynamical nonlinearity of the laser rate equations is described by the $I_{A}$ and $I_{B}$ terms. Hence, if the ideal modulating current waveform $I_{b c}(t)$ could be generated, the output optical waveform would not include any non-linear distortion and therefore could be easily equalized with common methods.

In implementations, the four sub-currents would be distorted by the linear electrical channel from the laser driver to the laser and by the parasitic electrical characteristics of the laser and associated packaging. Various methods could be used to correct this linear distortion: by design the channel distortion could be minimized, the laser driver could include a linear equalizer to correct the distortion, or the linear distortion could be included in the desired optical response and its effect corrected by an equalizer at the receiver. Since it is implementation dependent, for simplicity and clarity, we ignore this linear distortion and assume that it would be corrected by some method.

\section{B. ABCD approximation method}

From an implementation point of view, the generation of the sub-currents $I_{A}$ [eq. (10) or eq. (11)] and $I_{B}$ [eq. (9)] could be viewed as problematic due to the inclusion of the inverse of $N_{p}(t)$ [or $P(t)$ within the bracket to be differentiated in eq. (11)]. Therefore, a further approximation which does not involve the inversion of the desired target photon density of the output waveform is desirable. This is referred to as the "ABCD approximation" method. After inspection of the full set of equations for the back-calculation method, useful simplifications that reduce the implementation complexity at the transmitter in comparison to the full ABCD method can be made based on reasonable numerical approximations. Firstly, the sub-current $I_{B}(t)$ can be approximated by:

$$
I_{B}(t) \cong \widetilde{I}_{B}(t)=\frac{q V}{\Gamma} \cdot N_{p}^{\prime} \cdot\left[1+\frac{\varepsilon}{g_{0}} \cdot\left(\frac{1}{\tau_{p}}+\frac{1}{\tau_{e}}\right)\right]
$$

Secondly, from eq. (10), by omitting the higher order terms and approximating the inverse of $N_{p}(t)$ with the inverse of average photon density $\overline{N_{p}}$, the ideal $I_{A}(t)$ component can be approximated with the expression:

$$
I_{A}(t) \approx \widetilde{I}_{A}(t)=\frac{q V}{\Gamma g_{0}} \cdot\left(\frac{1}{\overline{N_{p}}}+\varepsilon\right) \cdot N_{p}^{\prime \prime}
$$

Using these approximations and given that $N_{p} \propto P(t)$ from eq. (1), it can be seen that $\widetilde{I_{B}}(t)$ and $\widetilde{I_{A}}(t)$ are proportional to the first and second derivative of the desired optical waveform: $\widetilde{I_{B}}(t) \propto \frac{d P(t)}{d t}, \widetilde{I_{A}}(t) \propto \frac{d^{2} P(t)}{d t^{2}}$. In addition, using the fact that $I_{C} \propto P(t)$, the ideal total back-calculated current $I_{b c}(t)$ can be approximated with the expression:

$$
I_{b c}(t) \approx \widetilde{I_{b c}}(t)=\underbrace{a \frac{d \widetilde{I_{B}}(t)}{d t}}_{=\widetilde{I_{A}}}+\underbrace{b \frac{d I_{C}(t)}{d t}}_{=\widetilde{I_{B}}}+I_{C}(t)+I_{D}
$$

The scaling parameters $a$ and $b$ are constants that dependent on the laser parameters and operating bias point and which can be found through the approximate expressions:

$$
\begin{aligned}
a \cong\left(\frac{1}{\overline{N_{p}}}+\varepsilon\right) \cdot \frac{g_{0} \tau_{e} \tau_{p}}{\left(\varepsilon+g_{0} \tau_{e}\right) \cdot\left(\varepsilon+g_{0} \tau_{p}\right)} \\
b \cong \tau_{p}+\frac{\varepsilon}{g_{0}}
\end{aligned}
$$

Eq. (14) is the core of the proposed ABCD approximation (and Stretched A) method. It stipulates that in order to generate the desired output waveform $P(t)$ and avoid nonlinear distortions, it suffices to derive the required linear component $I_{C}$ and its derivatives and estimate the scaling factors $a$ and $b$ in order to produce the approximate backcalculated current $\widetilde{I_{b c}}$. In the sections that follow, it is shown that, provided that the laser parameters are roughly known (either by design or measurement), (i) the alignment of the three current components $\widetilde{I_{A}}, \widetilde{I_{B}}$ and $I_{C}$ in time and (ii) the value of the scaling factors $a$ and $b$ have large tolerances making the scheme viable in real-world applications. In addition, various methods to implement this method in practice are suggested in Section IV. First however, a further improvement to the $\mathrm{ABCD}$ approximation method, named the Stretched A method, is proposed.

\section{Stretched A method}

Current and optical output waveforms for NRZ modulation based on eq. (12)-(14) were compared to those obtained from the full laser rate equations. It was noticed that whilst the $\mathrm{ABCD}$ approximation produced reasonable results, the accuracy could be improved if the $\widetilde{I}_{A}(t)$ component was appropriately shifted in time with respect to the rising and falling edges of the linear $I_{C}(t)$ component. In particular, a time advance needs to be introduced for positive transitions $(0 \rightarrow 1)$ while a reversed symmetric time delay is needed for the negative transitions $(1 \rightarrow 0)$. This waveform can be easily produced based on a version of $I_{C}(t)$ with an introduced amount of duty-cycle-distortion (DCD). The DCD stretches the duration of the periods of high current level and shrinks the duration of the periods of low current level of the waveform providing a time advance for the positive edges and time delay for the negative ones as required. This is illustrated in Fig. 1 and explains the sign convention used for the generated timing offset $d t$ throughout this work. The time axis of Fig. 1 is in units of unit intervals (UI), where a UI is equal to the NRZ symbol period, and this is used throughout this manuscript. This method for obtaining an improved approximation for the $I_{A}(t)$ subcurrent is named the Stretched A method and the resulting component denoted $I_{A}^{s t}(t)$. In the next section, the particular details of the method are explained and sample waveforms are illustrated. 


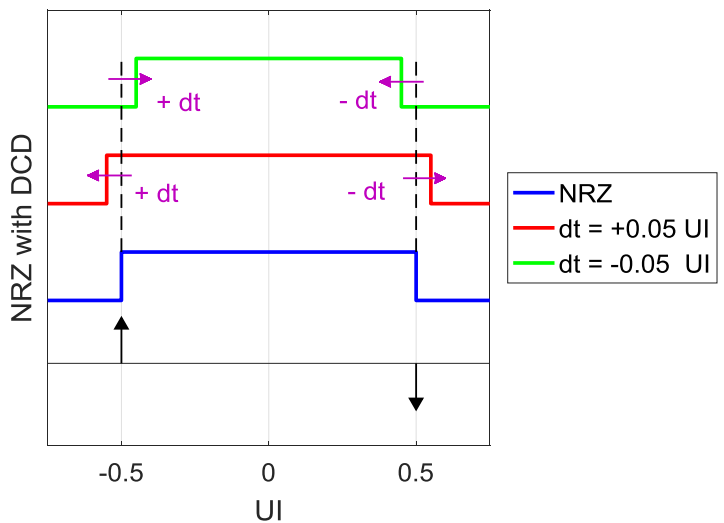

Fig. 1. Illustration of application of $\pm 10 \%$ DCD on a single NRZ pulse. The DCD generates an anti-symmetric timing offset $d t$ for the positive and negative edges of the pulse. The waveforms are offset in the $y$-axis for clarity.

\section{RESPONSES AND WAVEFORMS}

\section{A. Target optical waveform and VCSEL model}

To define the desired optical waveform, previous studies have used raised cosine (RC) responses, having roll-off factors in the range 0.1 to 1 as the basis for NRZ modulation $[15,16]$. In this study, we use the pulse response commonly employed to estimate the optical power budget for many 802.3 IEEE standards based on MMF and SMF links [35-39]. That pulse response, which we term the IEEE pulse response, is defined as the convolution of a Gaussian impulse with an ideal NRZ pulse. The Gaussian impulse response is defined by:

$$
h(t)=\frac{1}{\sigma \sqrt{2 \pi}} e^{-\frac{t^{2}}{2 \sigma^{2}}}
$$

where, $\sigma$ is the standard deviation of the response. The $10 \%$ to 90\% step response time $T_{c}$ due to eq.(17) is related to $\sigma$ with the expression $T_{c} \cong 2.563 \cdot \sigma$. In this paper, we will use the step response time $T_{c}$ as a parameter to define the desired optical waveform $P(t)$.

A single mode rate equation model of a VCSEL suitable for $25 \mathrm{~Gb} / \mathrm{s}$ operation is used as the basis for this study. Although $850 \mathrm{~nm}$ VCSELs are typically multimode devices, it has been shown that the single mode rate equations can provide an accurate description of their behaviour [40-42]. The rate equation parameters are stated in Table 1 . The values used here are not based on an actual physical device but are chosen to generate DC and high-frequency performance that match those expected of a typical $25 \mathrm{~Gb} / \mathrm{s}$ VCSEL. The simulated frequency response is shown in Fig. 2(a). To illustrate the modelled laser performance, the VCSEL is operated at close to typical conditions for high speed data transmission. The VCSEL is biased at $4.95 \mathrm{~mA}$ and modulated with a peak-topeak current of $4.1 \mathrm{~mA}$, which results in an optical modulation amplitude (OMA) of $6.2 \mathrm{dBm}$ and an extinction ratio (ER) of $4.6 \mathrm{~dB}$. Fig. 2 plots the deterministic, noise-free, eye diagrams calculated using the rate equation model at $25 \mathrm{~Gb} / \mathrm{s}$ and 50 $\mathrm{Gb} / \mathrm{s}$ for NRZ modulation and a pseudo-random binary sequence of length $2^{7}-1$ (PRBS-7). The associated electrical current modulation waveforms were created by convolving the IEEE pulse response with the PRBS. For this data rate, the step response time $T_{c}$ was set to $0.75 \times T$, where $T$ is the symbol period. The eye diagrams of Fig. 2(b) illustrate that the modelled laser is suitable for operation at $25 \mathrm{~Gb} / \mathrm{s}$. However, the obtained eye diagram at $50 \mathrm{~Gb} / \mathrm{s}$ [Fig. 2(c)] exhibits eye closure, skew, level thickness, increased overshoot and jitter which is due to the non-linearity of the laser.

TABLE I

VCSEL RATE EQUATION PARAMETERS

\begin{tabular}{|l|c|c|}
\hline \multicolumn{1}{|c|}{ Parameter } & Symbol & Value \\
\hline Mode (optical) confinement factor & $\Gamma$ & 0.18 \\
\hline Spontaneous emission factor & $\beta$ & 0.001 \\
\hline Active region volume & $V$ & $3.8 \times 10^{-18} \mathrm{~m}^{3}$ \\
\hline Electron lifetime & $\tau_{e}$ & $4.0 \times 10^{-9} \mathrm{~s}$ \\
\hline Photon lifetime & $\tau_{p}$ & $5.0 \times 10^{-12} \mathrm{~s}$ \\
\hline Gain slope constant & $g_{0}$ & $1.2 \times 10^{-11} \mathrm{~m}^{3} \mathrm{~s}^{-1}$ \\
\hline Gain compression factor & $\varepsilon$ & $4.125 \times 10^{-23} \mathrm{~m}^{3}$ \\
\hline Carrier density at transparency & $N_{0}$ & $5.0 \times 10^{24} \mathrm{~m}^{-3}$ \\
\hline \hline
\end{tabular}

(a)
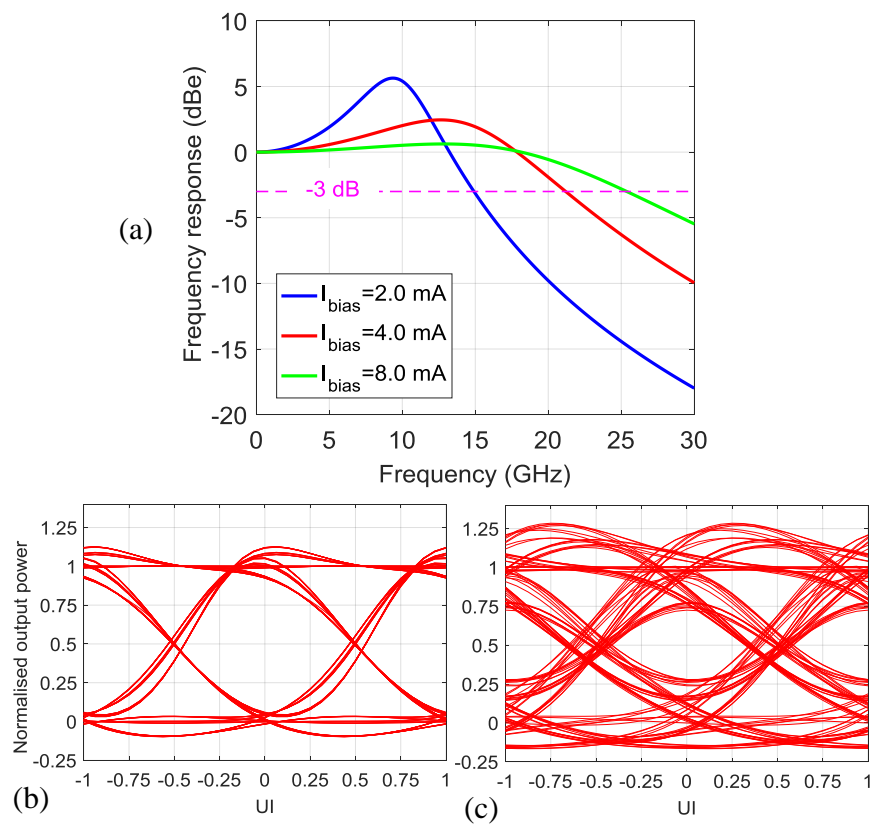

Fig. 2. (a) Simulated VCSEL frequency response with the rate equation model and eye diagrams for (b) $25 \mathrm{~Gb} / \mathrm{s}$ and (c) $50 \mathrm{~Gb} / \mathrm{s}$ NRZ transmission without non-linear correction for a $T_{c}$ of $0.75 \times T$.

\section{B. Step response by the $A B C D$ approximation method}

The proposed $A B C D$ approximation method is applied to correct the VCSEL non-linearity and considerably improve the output waveform to make it suitable for $50 \mathrm{~Gb} / \mathrm{s}$ transmission. Initially, the step response of the laser is studied to illustrate the shape of the required ideal sub-currents to generate a positive step in the output optical waveform $(0 \rightarrow 1$ transition) of the desired shape and amplitude. Assuming the 0 and 1 levels of the output waveform $P(t)$ correspond to photon densities $N_{p}^{L}$ and $N_{p}^{H}$ respectively via eq. (1) and that the transition happens at $t_{0}$, the target photon density $N_{p}(t)$ can be found by considering the desired impulse response $h(t)$ :

$$
N_{p}(t)=N_{p}^{L}+\left(N_{p}^{H}-N_{p}^{L}\right) \cdot \int_{-\infty}^{t} h\left(t-t_{0}\right) d t
$$

For the Gaussian impulse response used in this work and for $t_{0}=0, N_{p}(t)$ is found to be: 


$$
N_{p}(t)=N_{p}^{L}+\frac{N_{p}^{H}-N_{p}^{L}}{2} \cdot\left(1+\operatorname{erf}\left(\frac{t}{\sqrt{2} \sigma}\right)\right)
$$

As a result, the ideal four sub-currents $I_{A}, I_{B}, I_{C}$, and $I_{D}$ can be calculated from eq. (7)-(10). $I_{A}, I_{B}, I_{C}$, and the total ideal modulating current $I_{b c}$ for generating a step in the optical output waveform with a response time $T_{c}$ of $0.75 \times T$ and an amplitude similar to the one used for NRZ modulation at the two data rates of 25 and $50 \mathrm{~Gb} / \mathrm{s}$ are plotted in Fig. 3. As indicated above, the first two terms representing the $I_{A}$ and $I_{B}$ components are required to correct the dynamic non-linearity of the modelled laser. It can be noticed that their form is similar for the two data rates, but a larger magnitude is required for the higher data rate of $50 \mathrm{~Gb} / \mathrm{s}$ indicating larger laser non-linearity.
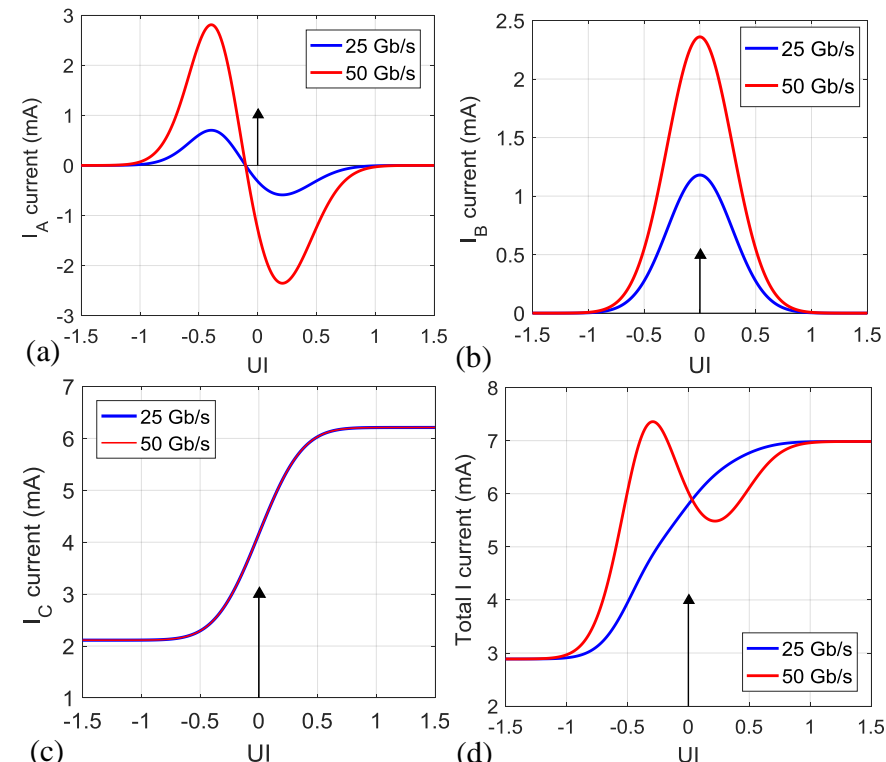

Fig. 3. Waveforms of ideal sub-currents (a) $I_{A}$, (b) $I_{B}$, (c) $I_{C}$ and total modulating current (d) $I_{b c}$ for a desired output step with $T_{c}$ of $0.75 \times T$ at 25 and $50 \mathrm{~Gb} / \mathrm{s}$. The term $I_{D}$ is constant and equal to the threshold current $(0.77 \mathrm{~mA})$. The black arrow indicates the timing of the step.

Using the proposed approximations, the $\widetilde{I_{A}}$ and $\widetilde{I_{B}}$ sub-currents can be obtained through eq. (12)-(13) and the total approximated ideal current $I_{s}^{+}(t)$ for a positive step input is obtained through the ABCD approximation method and eq. (14) and is given by:

$$
\begin{gathered}
I_{S}^{+}(t)= \\
=\underbrace{-\left(\frac{a \cdot b \cdot \Delta_{I}}{\sigma^{2}}\right) \cdot t \cdot h(t)}_{=\widetilde{I}_{A}}+\underbrace{b \cdot \Delta_{I} \cdot h(t)}_{=\widetilde{I}_{B}}+\underbrace{\left[\overline{I_{C}}+\frac{\Delta_{\mathrm{I}}}{2} \operatorname{erf}\left(\frac{t}{\sigma \sqrt{2}}\right)\right]}_{=I_{C}}+\underbrace{I_{t h}}_{=I_{D}}
\end{gathered}
$$

Here, $\bar{I}_{C}$ and $\Delta_{I}$ are the calculated mean and peak-to-peak amplitude of the $I_{C}$ component from eq. (8). Fig. 4 compares ideal $I_{A}$ and $I_{B}$ components from eq. (9) and (10) with the $\widetilde{I_{A}}$ and the $\widetilde{I_{B}}$ approximation described in eq. (12) and (13) for the step response at $50 \mathrm{~Gb} / \mathrm{s}$.

It can be observed that (i) the currents $I_{B}$ and $\widetilde{I_{B}}$ are practically identical, (ii) the $\widetilde{I}_{A}$ approximation has a similar shape as the ideal $I_{A}$ pulse but occurs at slightly different times. This is due to the omission of the higher order terms of eq. (10). Fig. 4(a) also plots the $I_{A}^{s t}$ pulse derived from the Stretched A method and the duty cycle distortion of the linear current component $I_{C}$. The time shifting of the $\widetilde{I_{A}}$ pulse produces a better approximation of the ideal current $I_{A}$.

(a)

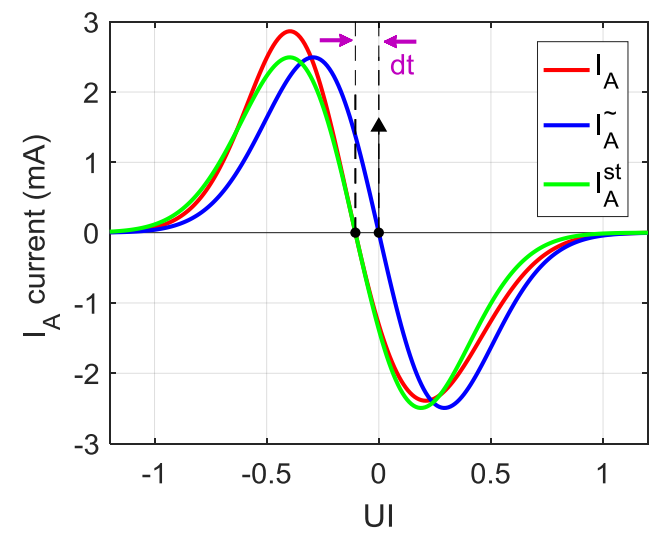

(b)

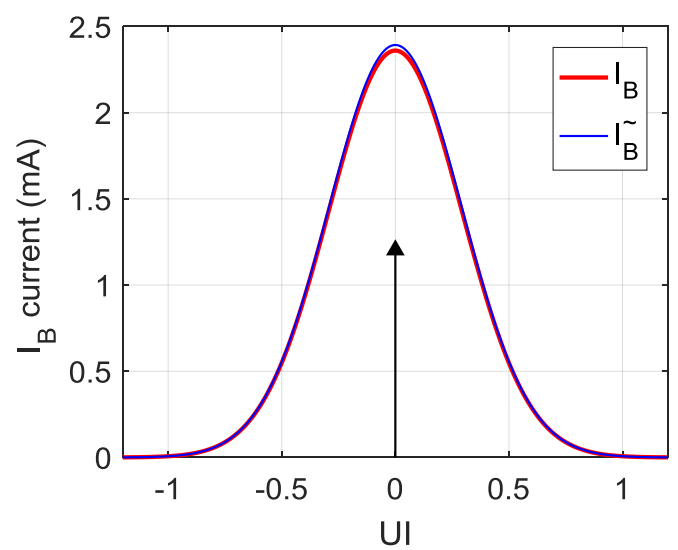

Fig. 4. Comparison of the (a) ideal $I_{A}$, approximate $\widetilde{I_{A}}$ and $I_{A}^{s t}$ (timeshifted version of $\widetilde{I_{A}}$ ) and (b) ideal $I_{B}$ and approximate $\widetilde{I_{B}}$ for a positive step in $I_{C}(0 \rightarrow 1$ transition $)$.

A symmetric expression can be derived for the total approximated ideal current $I_{s}^{-}(t)$ required to generate a negative step $(1 \rightarrow 0$ transition $)$ of the same shape and amplitude:

$$
\begin{gathered}
I_{S}^{-}(t)= \\
=\underbrace{\left(\frac{a \cdot b \cdot \Delta_{I}}{\sigma^{2}}\right) \cdot t \cdot h(t)}_{=\widetilde{I}_{A}}+\underbrace{\left(-b \cdot \Delta_{I} \cdot h(t)\right)}_{=\widetilde{I}_{B}}+\underbrace{\left[\overline{I_{C}}-\frac{\Delta_{\mathrm{I}}}{2} \operatorname{erf}\left(\frac{t}{\sigma \sqrt{2}}\right)\right]}_{=I_{C}}+\underbrace{I_{t h}}_{=I_{D}}
\end{gathered}
$$

As a result, the total back-calculated current $\widetilde{I_{b c}}(t)$ can be written as the summation of the current required to generate the desired positive and negative steps at the respective times $t_{i}^{+}$and $t_{i}^{-}$:

$$
\widetilde{I_{b c}}(t)=\sum_{\mathrm{i}} I_{s}^{+}\left(t-t_{i}^{+}\right)+\sum_{\mathrm{i}} I_{s}^{-}\left(t-t_{i}^{-}\right)
$$

\section{Results at $50 \mathrm{~Gb} / \mathrm{s}$ for the $\mathrm{ABCD}$ approximation method}

Combining the corresponding four sub-currents, the ideal and approximate back-calculated modulating currents $I_{b c}(t)$ and $\widetilde{I_{b c}}(t)$ are calculated for the PRBS-7 input at $50 \mathrm{~Gb} / \mathrm{s}$ with a desired response time $T_{c}$ of $0.75 \times T$ for the same operating conditions as the ones used for Fig. 2. Fig. 5 illustrates the waveforms of the ideal sub-currents and total back-calculated modulating current as well as the resulting (i.e. target) optical output waveform $P_{\text {out }}(t)$. For clarity, the first 60 bits of the 
(a)

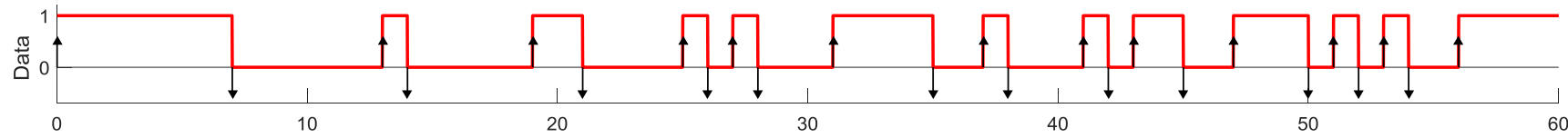

(b)

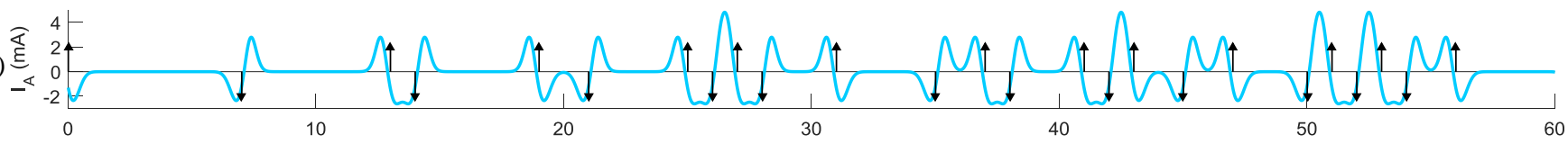

(c)

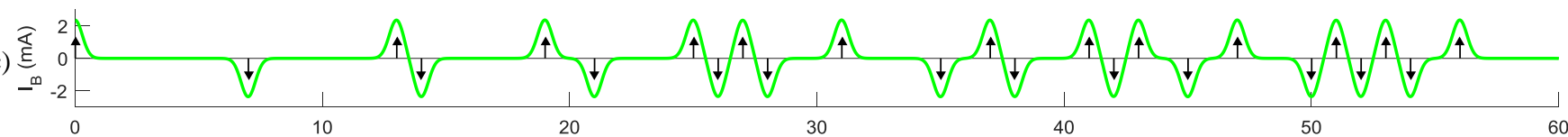

(d)

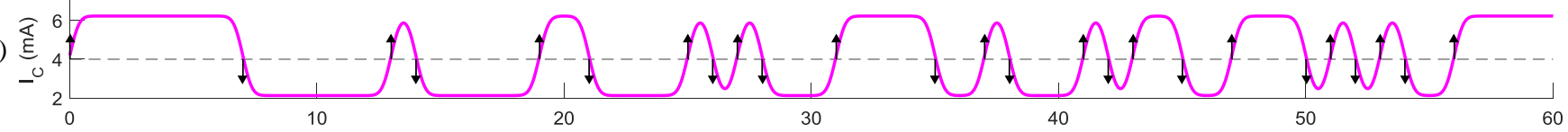

(e)

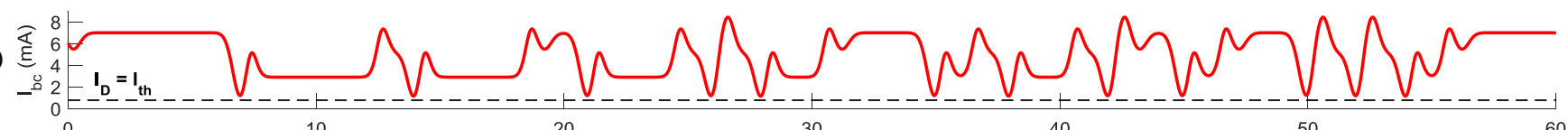

(f)

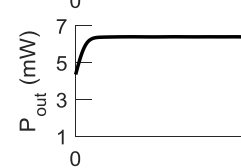

10

20

40

व $\begin{array}{r}3 \\ 0\end{array}$

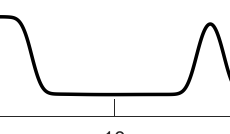

10

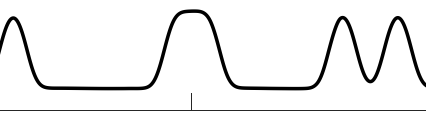

20

UI

Fig. 5. (a) Data input with transitions noted for the first 60 bits of the PRBS-7 pattern and (b-f) waveforms of the ideal sub-currents (b) $I_{A}$, (c) $I_{B}$ and (d) $I_{c}$ and (e) total back-calculated modulating current $I_{b c}$ and (f) output optical waveform $P(t)$ for a PRBS-7 input at $50 \mathrm{~Gb} / \mathrm{s}$ and desired response time $T_{c}$ of $0.75 \times T$.

transmitted PRBS-7 pattern are shown as these contain most of the transition types, including long sequences of $1 \mathrm{~s}$ and $0 \mathrm{~s}$, an isolated one, and alternating single 1s and 0s. Fig. 6 shows the corresponding eye diagram and related metrics: eye height $h$, width $w$, and effective eye area metric $S$, defined as $S=w \times h$, for the complete PRBS-7 pattern. The combination of these eye diagram metrics and the root mean squared error (RMSE) of the normalised output waveform with respect to the normalised target (ideal) waveform are employed to assess the quality of the obtained optical waveforms with the proposed approximation methods and therefore, their effectiveness in reducing the non-linear distortions. For reference, the values of the eye diagram metrics for the conventional NRZ modulation (non-corrected waveform) [Fig. 2(c)] are calculated and are found to be: $h=0.447, w=0.730, S=0.326, \operatorname{RMSE}=0.111$.

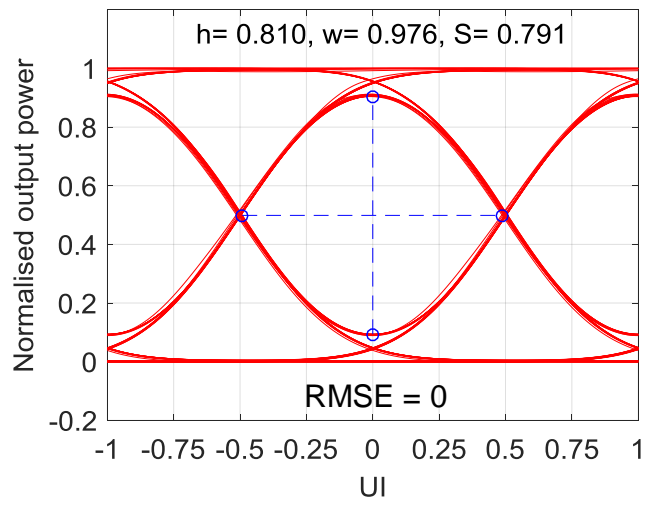

Fig. 6. Eye diagram at $50 \mathrm{~Gb} / \mathrm{s}$ for the ideal back-calculated modulating current $I_{b c}$ with related metrics noted.
Applying the ABCD approximation method and eq. (12) and (13), the approximate sub-currents $\widetilde{I_{A}}$ and $\widetilde{I_{B}}$ and corresponding total modulating currents $\widetilde{I_{b c}}(t)$ are calculated for the same data transmission parameters and desired response time $T_{c}$ of $0.75 \times T$. Fig. 7 illustrates the corresponding eye diagram of the output optical waveform with the eye diagram metrics and RMSE value noted.

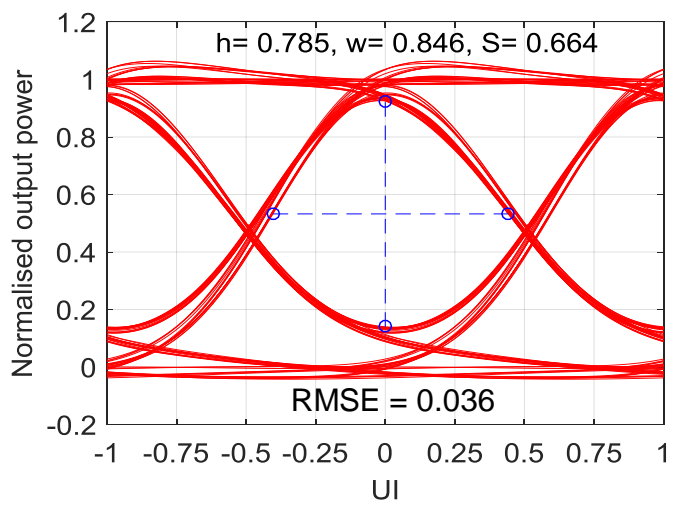

Fig. 7. Eye diagram at $50 \mathrm{~Gb} / \mathrm{s}$ for the approximate total backcalculated modulating current $\widetilde{I_{b c}}$ with eye metrics noted.

It can be clearly noticed that the ABCD approximation method greatly improves the obtained eye diagram in comparison with that obtained for the conventional NRZ modulation at $50 \mathrm{~Gb} / \mathrm{s}$ [Fig. 2(c)]. This is reflected in the greatly improved values of the eye metrics and the RMSE value. However, it can be noticed that there still exists some non-linear distortion in the optical waveform with some eye skew, jitter, overshoot and level thickness which results in reduced eye metrics in comparison to those obtained for the ideal modulating current 
(a)

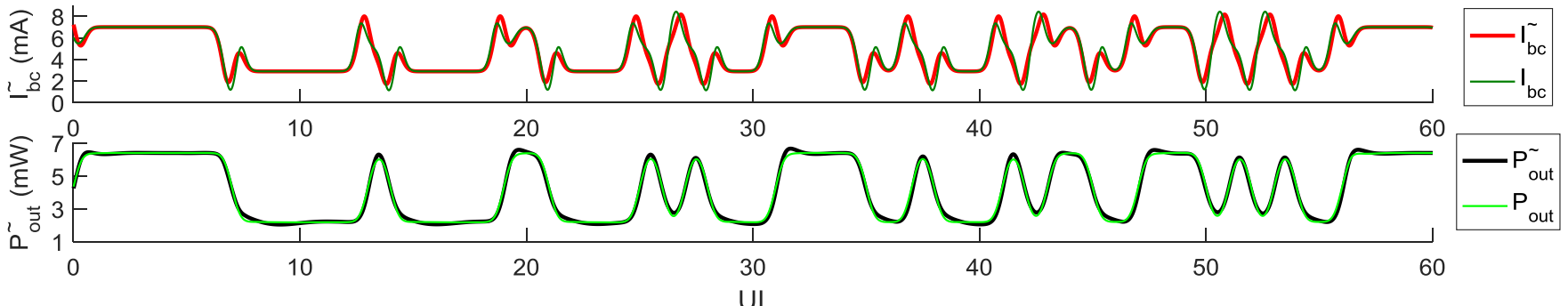

Fig. 8. Waveforms of (a) the total back-calculated modulating current $\widetilde{I_{b c}}$ obtained with the ABCD approximation method and (b) the resulting output optical waveform $\widetilde{P_{\text {out }}}$ for a PRBS-7 input (first 60 bits shown) at $50 \mathrm{~Gb} / \mathrm{s}$ and desired response time $T_{c}$ of $0.75 \times T$. The respective ideal back-calculated waveforms $I_{b c}$ and $P_{o u t}$ are also shown in green for comparison.

(Fig. 6). Fig. 8 shows the waveforms of the approximate total modulating current $\widetilde{I_{b c}}$ and the generated output waveform. The respective ideal waveforms are also shown (green lines) for comparison.

\section{STRETCHED A METHOD AND TOLERANCES}

\section{A. Sensitivity analysis for $I_{A}^{s t}$ and $\widetilde{I_{B}}$}

As indicated in the previous section, by applying the Stretched A method, a better approximation $I_{A}^{s t}$ of the ideal $I_{A}$ is obtained [Fig. 4(a)]. $I_{A}^{s t}$ can be obtained from the $\widetilde{I_{A}}$ pulse by time shifting and amplitude scaling:

$$
I_{A}^{s t}(t)=\gamma \cdot \widetilde{I_{A}}(t+d t)
$$

For a relative amplitude $\gamma=1$ and timing offset $d t=0$, the $I_{A}^{s t}$ pulse is the same as $\widetilde{I_{A}}$. The optimum parameters of this new approximate pulse $I_{A}^{s t}$ and their tolerances are investigated by assessing the resemblance of the generated output optical waveforms to the target (ideal) waveform (RMSE value) and comparing their eye diagram metrics $(h, w, S)$. The total modulating current is obtained for different values of the $\gamma$ and $d t$ parameters and the resulting output optical waveform is calculated for $50 \mathrm{~Gb} / \mathrm{s}$ data transmission and the same operating conditions previously stated. The RMSE of the output waveform and the corresponding eye diagram metrics are obtained for each set of parameters studied. It should be noted that for these simulations (i) mainly positive offsets $d t$ are considered as the ideal $I_{A}$ current has a time advance (for a $0 \rightarrow 1$ transition) with respect to $\widetilde{I}_{A}$ [Fig. 4(a)], (ii) the timing offset is assumed to be reversed for the negative transition $(1 \rightarrow 0)$ as this is what the DCD of the generating NRZ waveform would produce (Fig. 1) and (iii) the remaining three sub-currents are assumed to be the approximate $\widetilde{I_{B}}$ and ideal $I_{C}$ and $I_{D}$ components.

Fig. 9 shows contour plots of the obtained eye height $(h)$, width $(w)$ and effective area $(S=w \times h)$ while Fig. 10 the corresponding RMSE value as a function of the two parameters $\gamma$ and $d t$ of $I_{A}^{s t}$. There is a broad region where the Stretched A method performs as well as or better than the $\widetilde{I}_{A}$ approximation obtained with the ABCD approximation method of eq. (14). The values obtained for the $\widetilde{I}_{A}$ approximation are indicated in the plots with the cyan dot $(\gamma=1, d t=0)$ and the $\widetilde{I_{A}}$ notation. The set of parameters yielding the maximum eye area is found to be $\gamma \sim 1.07, d t \sim 0.08$ UI. This point is noted in Fig. 9(c) with a yellow dot and the notation $I_{A}^{s t, o p t}$. It coincides with the global minimum for the RMSE values (Fig. 10) indicating the agreement of the two metrics. Fig. 11 compares the waveforms of the ideal $I_{A}$, approximate $\widetilde{I}_{A}$ and optimised $I_{A}^{s t}$ per Fig. 10(c) and shows the resulting eye diagram at 50 $\mathrm{Gb} / \mathrm{s}$ for the optimised $I_{A}^{s t}$ with its metrics noted. Clearly the Stretched A method produces an output waveform very close to the target one (Fig. 6) with little residual non-linearity (eye skew, level thickness, overshoot and jitter) and eye metrics very close to those obtained from the target waveform. This is explained by Fig. 11(a) where it can be seen that the shape of the $I_{A}^{s t}$ pulse (and in particular the rising and falling edges) approximates very well the ideal pulse $I_{A}$. In addition, the eye area $S$ and RMSE contour plots [ Fig. 9(c) and Fig. 10] indicate the method has relatively large tolerances with good performance obtained (large $S$ values and small RMSE values) for a wide range of the $\gamma$ and $d t$ parameters.

To further demonstrate that the optical output waveforms obtained through the Stretched A method have very low nonlinearity, a linear equalizer is applied to fully open the eye diagrams. An 11-tap T-spaced feedforward equalizer (FFE) is applied on the output optical waveforms obtained for the different combinations of the $\gamma$ and $d t$ parameters. Eleven taps were used as it was found that more taps did not significantly improve the quality of the waveforms for the non-corrected or corrected cases. The eye metrics $(h, w, S)$ of the equalized waveforms as well as the noise enhancement factor (NEF) introduced by the equalizer are calculated and plotted in Fig. 12. It can be clearly seen that the FFE can successfully equalize the generated optical waveforms and produce more open eye diagrams with $h \geq 0.9$ and $w \geq 0.8 \mathrm{UI}$ (or equivalently $S \geq 0.7$ ) for a broad range of amplitude scaling factors $\gamma$ and timing offsets $d t$. The NEF for these parameters is small ( $\leq 0.6 \mathrm{~dB}$ for the great majority) and very close to the value obtained for the ideal $I_{A}(\mathrm{NEF}=0.45 \mathrm{~dB})$ indicating that the waveforms produced through the $\mathrm{ABCD}$ approximation and in particular, the Stretched A method, feature very little non-linear distortion. The best performance in terms of eye metrics is again obtained for the set of parameters for $I_{A}^{s t, o p t}$ identified above: $\gamma \sim 1.07, d t \sim 0.08 \mathrm{UI}$ [point $I_{A}^{s t, o p t}$ in Fig. 12]. The eye diagrams obtained after equalization with the 11-tap FFE of the optical waveform generated with conventional modulation without any non-linear correction [Fig. 2(c)] and that using the ABCD method when the ideal $I_{A}$ (Fig. 6), approximate $\widetilde{I}_{A}$ (Fig. 7) and optimised $I_{A}^{\text {st,op }}$ [Fig. 11(c)] are employed, are shown in Fig. 13. It can be clearly 
seen that the non-corrected equalized eye diagram [Fig. 13(a)] exhibits significant level thickness, overshoot, jitter and greatly reduced effective area. In addition, the eye diagram of the equalized waveform obtained with the Stretched A method [Fig. 13(d)] is similar to the ideal one [Fig. 13(b)] with similar eye metrics too.

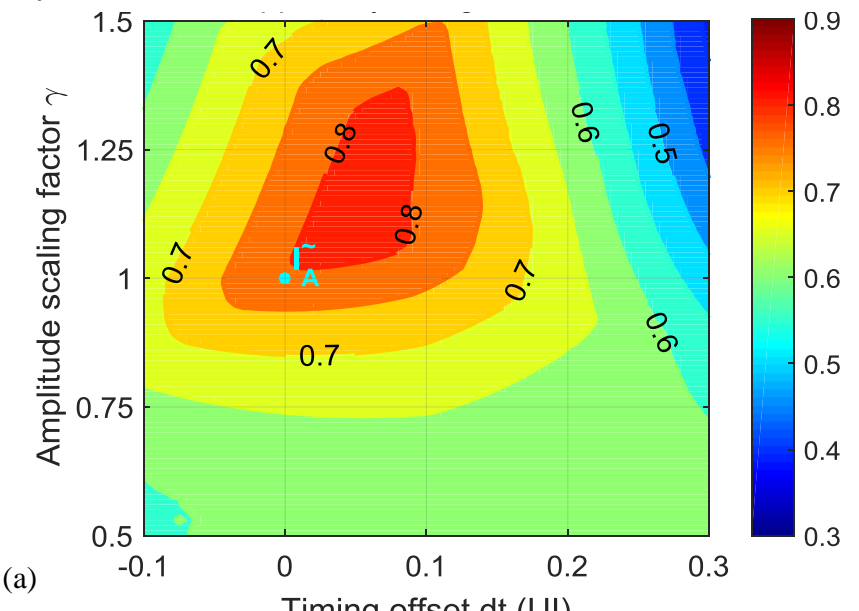

(a)
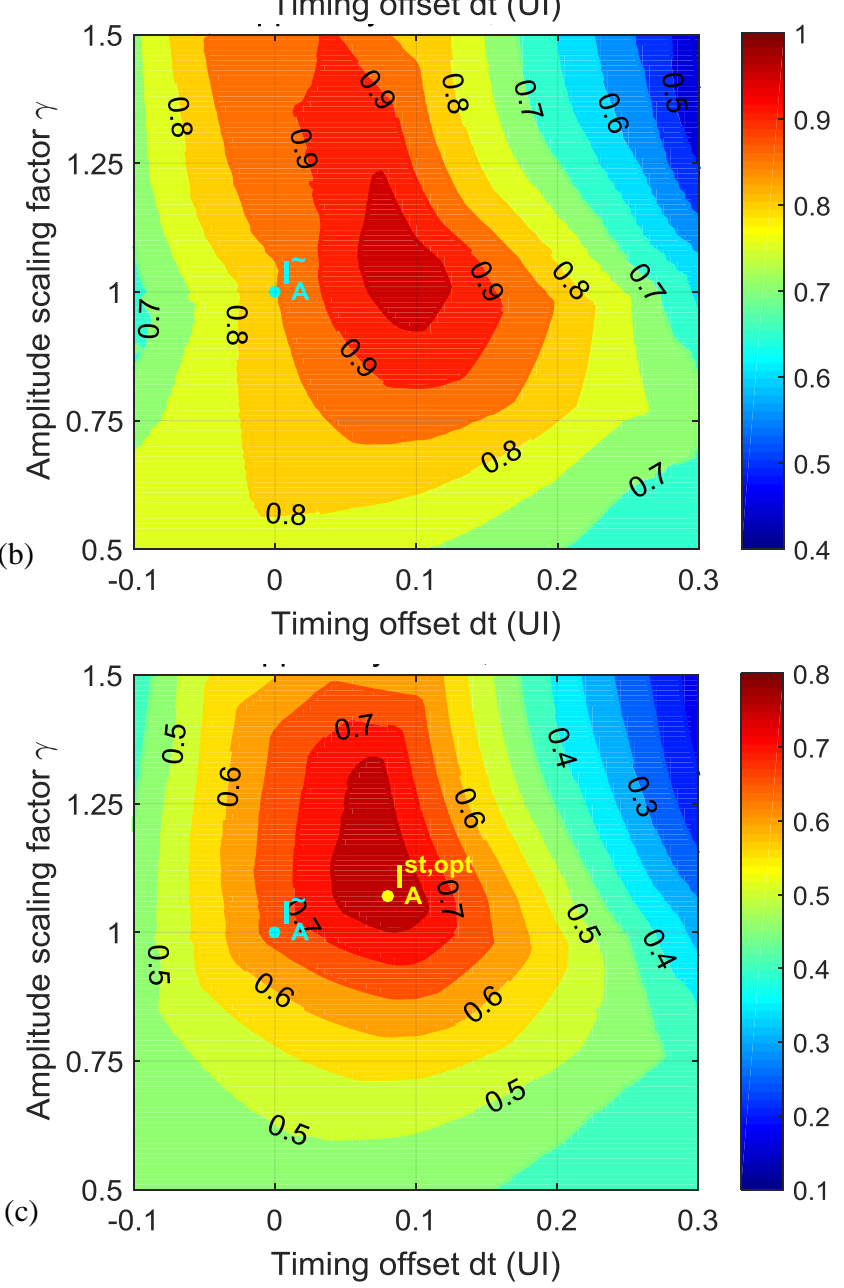

Fig. 9. Eye diagram metrics: (a) height $h$, (b) width $w$ and (c) area $S$ as a function of the $I_{A}^{s t}$ parameters: relative amplitude $\gamma$ and timing offset $d t$ for $50 \mathrm{~Gb} / \mathrm{s}$ data transmission for the Stretched A method.

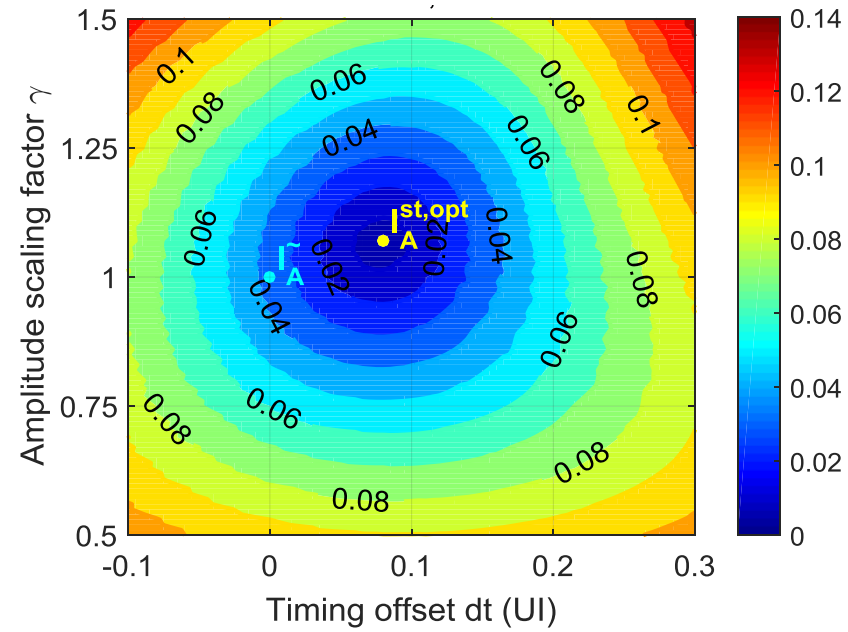

Fig. 10. RMSE value of the output optical waveform as a function of the $I_{A}^{s t}$ parameters: relative amplitude $\gamma$ and timing offset $d t$ for 50 $\mathrm{Gb} / \mathrm{s}$ data transmission for the Stretched A method.

(a)
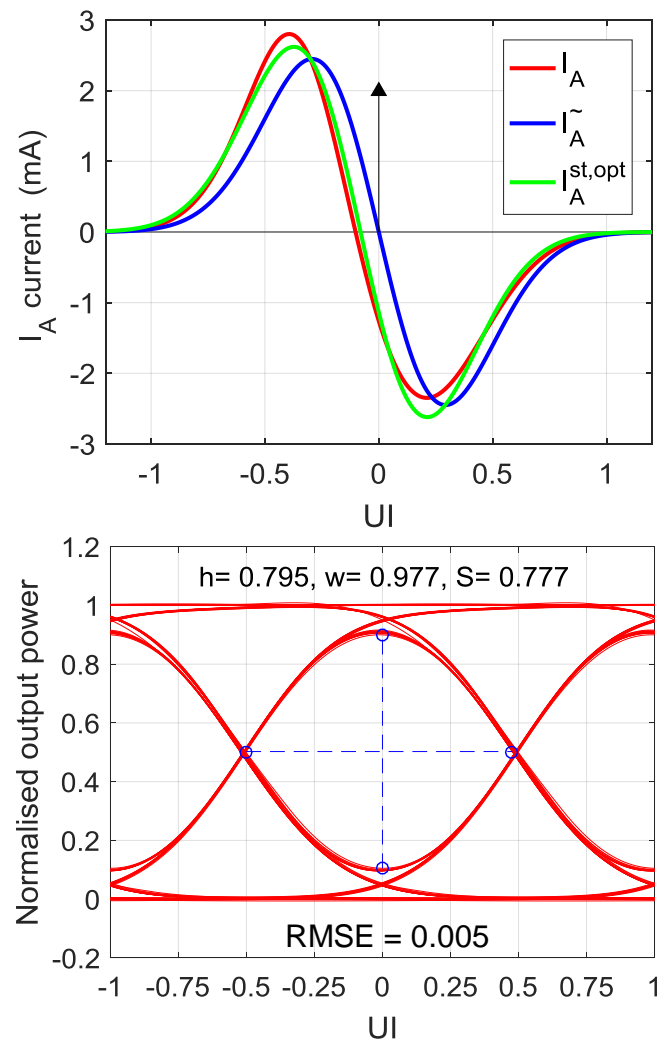

Fig. 11. (a) Comparison of the ideal $I_{A}$, approximate $\widetilde{I_{A}}$ and optimised $I_{A}^{\text {st,opt }}$ pulse and (b) eye diagram at $50 \mathrm{~Gb} / \mathrm{s}$ with the relevant metrics noted when the optimised $I_{A}^{\text {st,opt }}$ sub-current is used. 
(a)

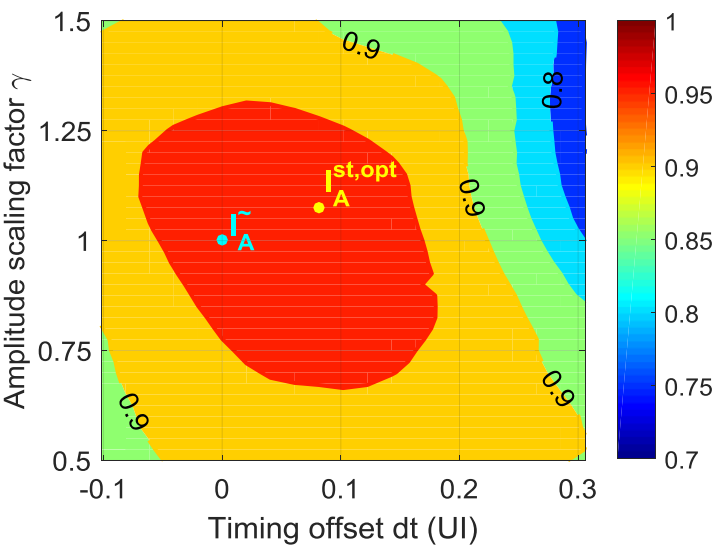

(b)
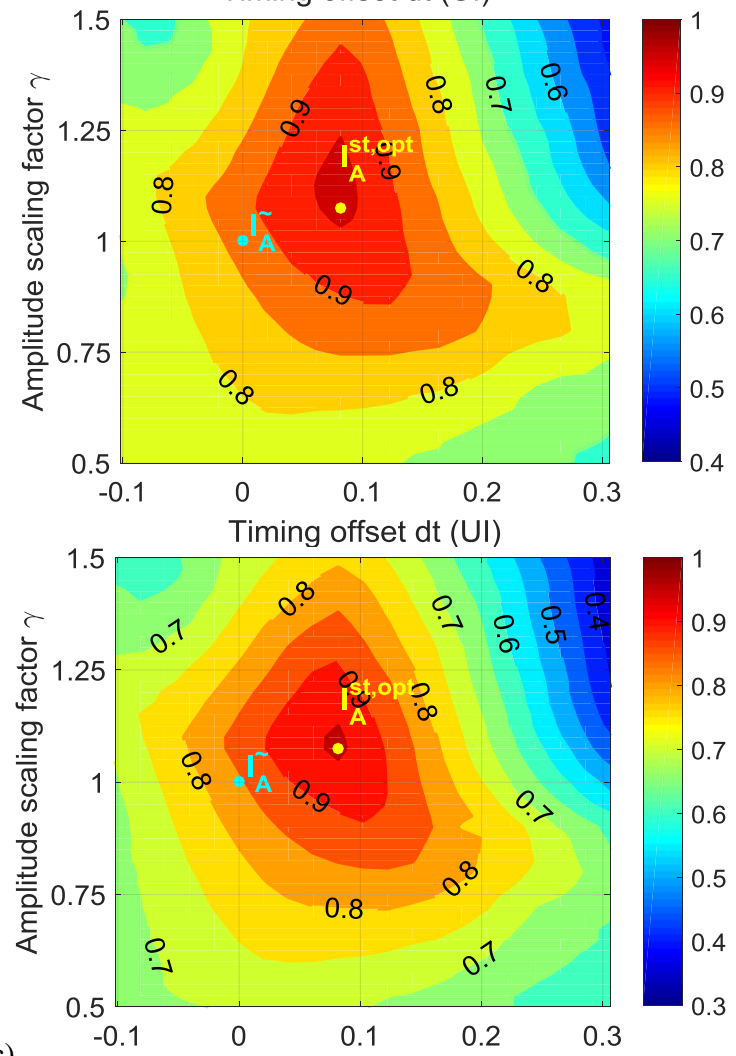

(c)

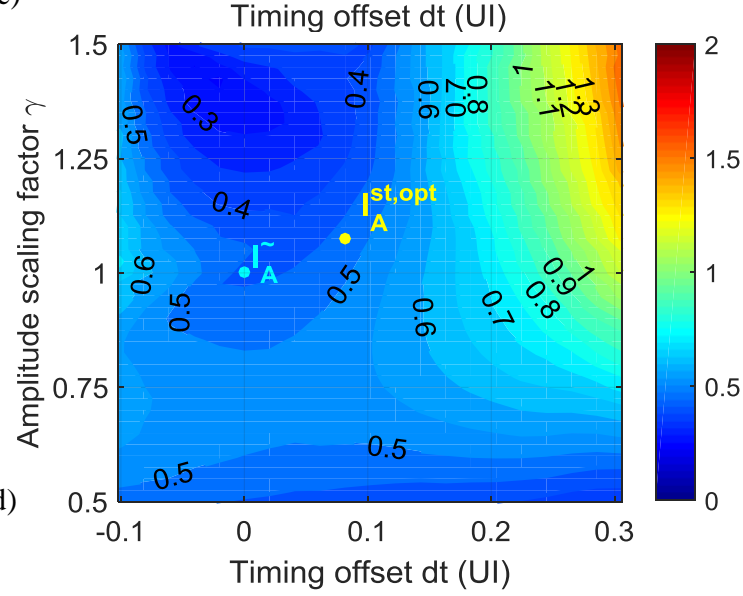

Fig. 12. Contour plots of eye metrics: (a) height $h$, (b) width $w$ and (c) area $S$ after the application of the T-spaced 11-tap FFE on the 50 $\mathrm{Gb} / \mathrm{s}$ optical waveforms obtained through the Stretched A method and (d) noise enhancement factor (in $\mathrm{dB}$ ) as a function of the $I_{A}^{S t}$ parameters: relative amplitude $\gamma$ and timing offset $d t$.
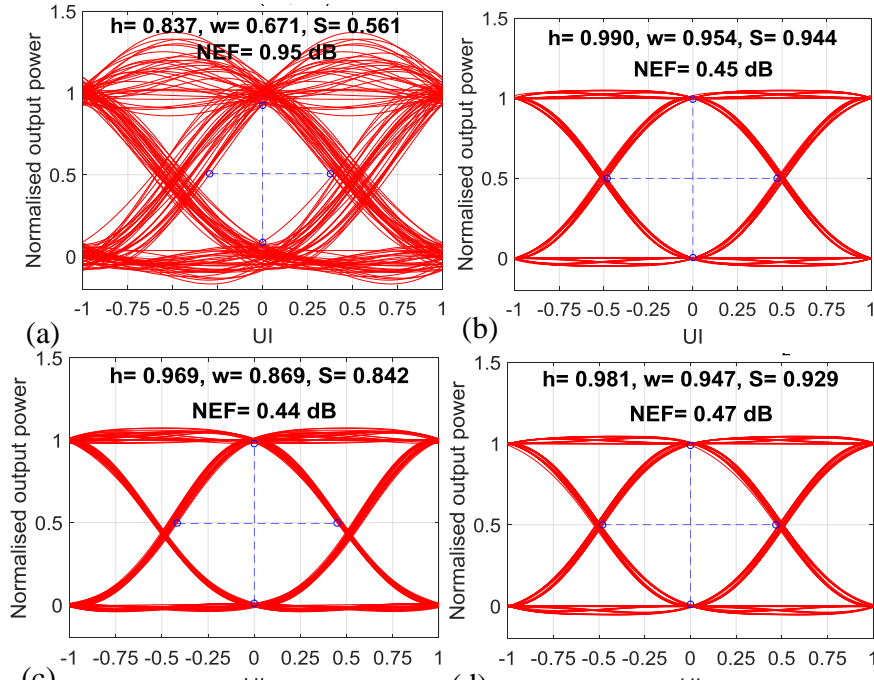

(b)

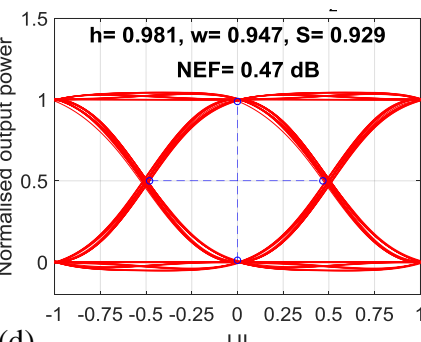

(d)

Fig. 13. Equalized eye diagrams at $50 \mathrm{~Gb} / \mathrm{s}$ and $T_{C}=0.75 \times T$ with the 11-tap FFE for (a) the conventional NRZ modulating signal, (b) the full $\mathrm{ABCD}$ method (ideal $I_{A}$ ), (c) the ABCD approximation method $\left(\widetilde{I_{A}}\right)$ and $(\mathrm{d})$ the Stretched A method (optimised $I_{A}^{s t, o p t}$ ).

A similar sensitivity analysis has been carried out for $\widetilde{I_{B}}(t)$ for $50 \mathrm{~Gb} / \mathrm{s}$ PRBS-7 transmission and for a desired step response time $T_{C}$ of $0.75 \times T$. The approximate $\widetilde{I_{B}}$ pulse is offset in time and scaled in amplitude:

$$
\widetilde{I_{B}^{o f f}}(t)=\delta \cdot \widetilde{I_{B}}\left(t+d t_{B}\right)
$$

and the RMSE of the resulting output waveform $\widetilde{P_{\text {out }}}$ with respect to the ideal (i.e. target) waveform $P_{\text {out }}$ is obtained. For the simulations, it is assumed that the optimised sub-current $I_{A}^{\text {st,opt }}$ obtained through the Stretched A method and the ideal $I_{C}$ and $I_{D}$ sub-currents are employed. Fig. 14 shows the obtained RMSE as a function of the parameters $\delta$ and $d t_{B}$.

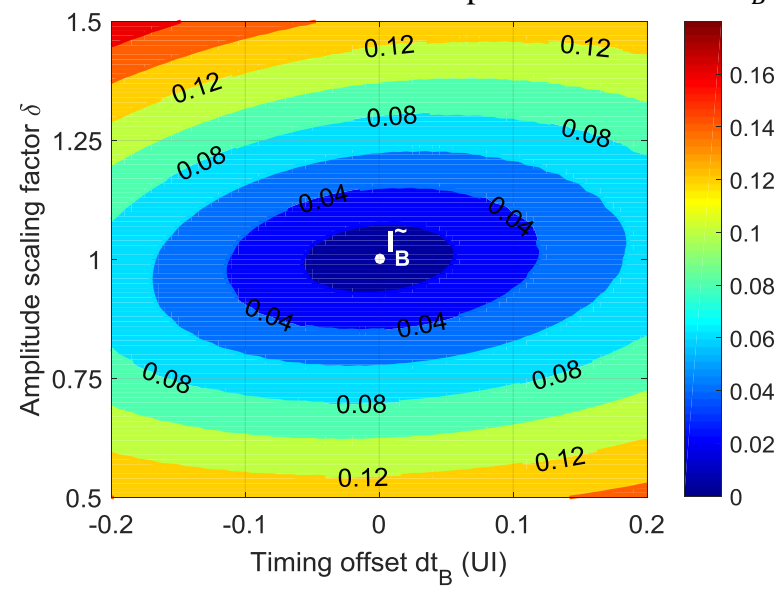

Fig. 14. RMSE of the output waveform as a function of the scaling parameter $\delta$ and timing offset $d t_{B}$ for the sub-current $I_{B}^{\widetilde{o f f}}(t)$.

As expected, the optimum performance is achieved for $\delta=1$ and $d t=0 \mathrm{UI}$, as the $\widetilde{I_{B}}$ approximation is very close to the ideal $I_{B}$ sub-current [Fig. 4(b)]. The plot demonstrates relaxed alignment tolerances for the generation of $\widetilde{I_{B}}$, with good performance obtained (RMSE $\leq 0.05)$ for a wide range of timing offsets, larger than $\pm 10 \%$ of the bit period and nominal amplitude. 


\section{B. Tolerance to timing offset and adaptive gain scheme}

In practical implementations, the $I_{A}^{s t}$ waveform created through the Stretched A method from $I_{C}$, would need to be realigned to the $\widetilde{I}_{B}$ and $I_{C}$ waveforms. To investigate the effect of misalignment of $I_{A}^{s t}$ on the generated optical waveform, the RMSE of the laser output waveform was calculated as a function of the relative delay $t_{o f f}$ of $I_{A}^{s t}$ and the rising/falling edge of the perfectly aligned $\widetilde{I_{B}}(t)$ and $I_{C}(t)$ waveforms. This time shift, in contrast to the one studied in section III.C (Fig. $1)$, results in the same type of delay for both the positive and negative transitions $(0 \rightarrow 1$ and $1 \rightarrow 0)$ (Fig. 15). Therefore, it results in different form of the total modulating current than the one studied in the previous section.

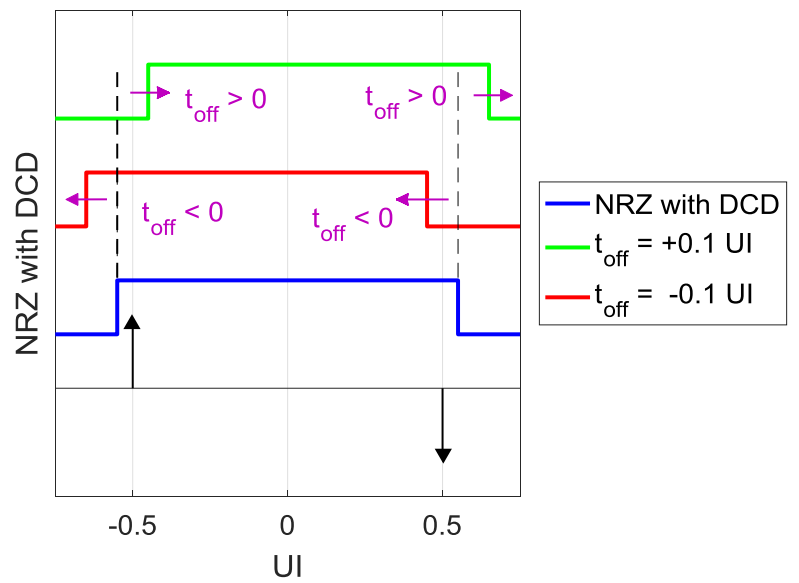

Fig. 15. Illustration of timing offset in the alignment of the $I_{A}^{S t}$ pulses generated via the Stretched A method. The $\widetilde{I_{B}}$ and $I_{C}$ pulses are assumed to be perfectly aligned with the edge of each transition (edges shown here for the transmission of a single $1 \mathrm{bit}$ ).

The RMSE value of the output waveform obtained via the Stretched A method for PRBS-7 transmission at $50 \mathrm{~Gb} / \mathrm{s}$ and a desired step response time $T_{C}$ of $0.75 \times T$ is calculated for different values of the timing misalignment $t_{o f f}$ and is plotted in Fig. 16. The RMSE value obtained for the conventional NRZ modulation [un-corrected waveform, Fig. 2(c)] is also shown for reference. The parameters of the Stretched A method used here are: $\gamma=1.07$ and $d t=0.08 \mathrm{UI}$ for $I_{A}^{s t}(t)$. The plot indicates that a relatively large tolerance to achieve RMSE values $\leq 0.05$ of $\sim \pm 0.07$ UI to this type of misalignment is obtained. It should be noted that misalignment beyond $\sim 0.1 \mathrm{UI}$ results in larger vertical eye openings, as the $I_{A}^{s t}$ pulse operates essentially as a frequency booster (equalizer), without correcting all of the non-linear signal distortion, as is evidenced by the increased jitter and level thickness of the eye diagrams in this region (Fig. 16).

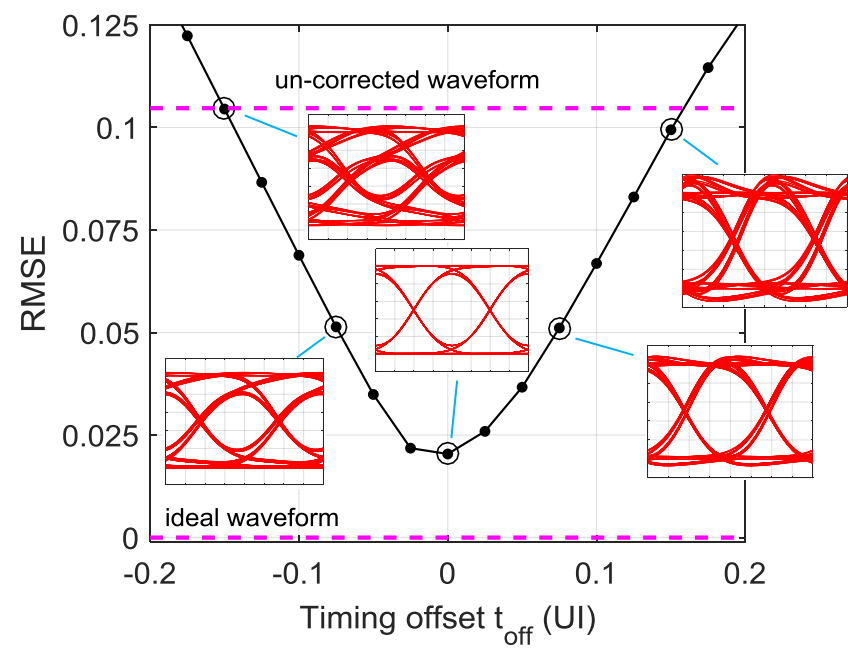

Fig. 16. RMSE for the output waveform obtained through the Stretched A method at $50 \mathrm{~Gb} / \mathrm{s}$ and a desired step response time $T_{C}=0.75 \times T$ as a function on the timing offset $t_{\text {off }}$ and the respective eye diagrams for a selection of offsets. Dashed lines indicate the RMSE value obtained for the ideal and un-corrected waveform.

It was found that an improved tolerance to the timing misalignment $t_{o f f}$ was enabled by varying the amplitude of the $I_{A}^{s t}(t)$ and $\widetilde{I_{B}}(t)$ currents as a function of the timing offset $t_{\text {off }}$. Inspection of the total current waveforms, not shown herein, indicated that in the region of low RMSEs the different combinations of the relative gains for $I_{A}^{s t}(t)$ and $\widetilde{I_{B}}(t)$ produced similar total modulation current waveforms that approximate the ideal back-calculated modulating current $I_{b c}(t)$. This can be achieved in practice by adaptively changing the gain parameters $a$ and $b$ in eq. (14). Their optimum values for each timing offset $t_{\text {off }}$ are found by minimising the RMSE of the output waveform with respect to the target (i.e. ideal) waveform. The scheme is applied for the same data transmission parameters $(50 \mathrm{~Gb} / \mathrm{s}, \mathrm{PRBS}-7)$ and Stretched A method implementation $\left(T_{C}=0.75 \times T, d t=\right.$ $0.08 \mathrm{UI}$ ) and the obtained results are shown in Fig. 17.

The observed variation in the optimum values of $a$ and $b$ can be explained as follows. As $t_{o f f}$ is made more negative, the scaling factors for $I_{A}^{s t}(t)$ and $\widetilde{I}_{B}(t)$ both increase [Fig. 17(a)]. In this region the output optical waveforms tend to be approximately linear however the eye opening systematically reduces and hence the eye metrics degrade [Fig. 17(b)]. This behaviour is due to a portion of the various currents acting as a form of low pass filter due to the phase mismatch between them. At some point the $I_{A}^{s t}(t)$ and $\widetilde{I_{B}}(t)$ currents would become excessive and/or the eye opening would become impractically small.

As $t_{o f f}$ is made more positive, the scaling factors reduce. However, for $t_{o f f}$ values in the range $\sim 0$ to $0.6 \mathrm{UI}$, the scaling factor $a$ for $I_{A}^{s t}(t)$ decreases much more slowly than the scaling factor $b$ for $\widetilde{I_{B}}(t)$ [Fig. 17(a)]. In this region, a portion of the currents is acting as a form of linear frequency "boosting" filter which partially equalises the output optical waveform increasing the eye opening and maintaining or improving the eye metrics [Fig. 17(b)]. Once the scaling factor 
for $\widetilde{I_{B}}(t)$ becomes zero $\left(t_{o f f}>0.6 \mathrm{UI}\right)$, the scaling factor for $I_{A}^{s t}(t)$ reduces more quickly. In this region there is no correction for the non-linearity. However, the $I_{A}^{s t}(t)$ current creates a high frequency boost effect which, to some extent, equalises the optical output waveform.

(a)
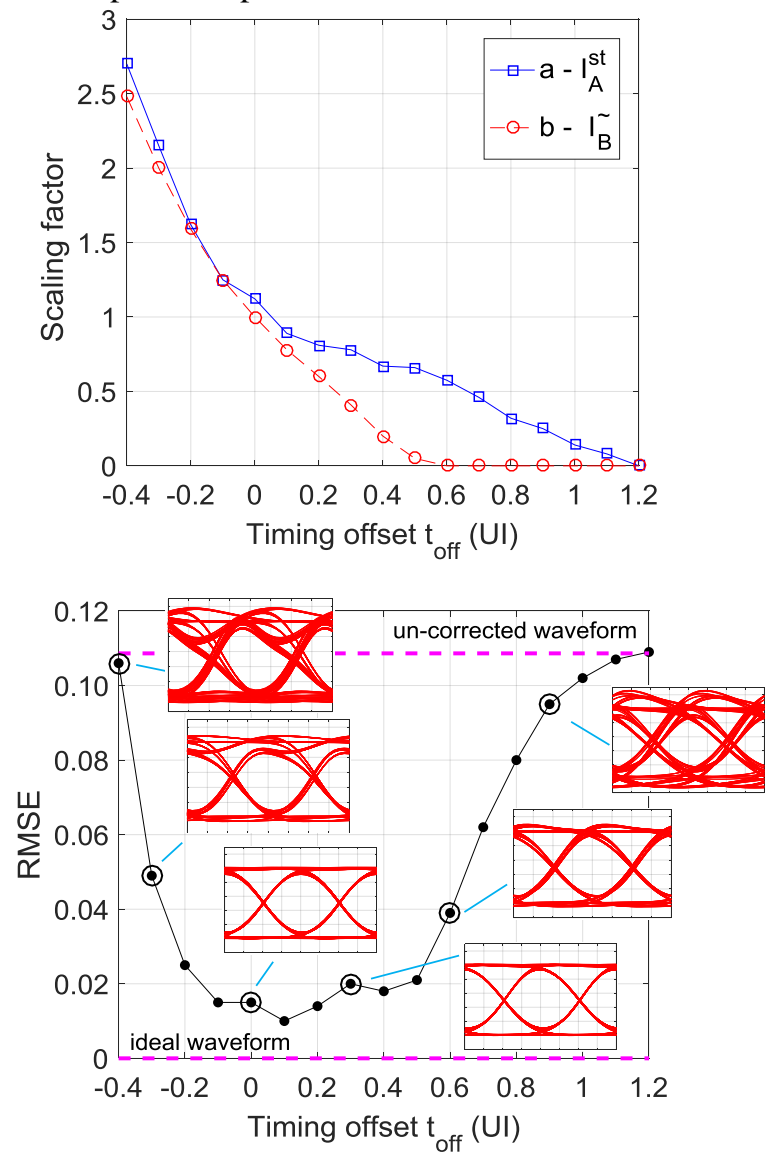

Fig. 17. (a) Optimized scaling factors $\alpha$ and $b$ as a function of the time offset $t_{\text {off }}$ when the adaptive gain scheme is applied on the $I_{A}^{S t}(t)$ and $\widetilde{I_{B}}(t)$ components at $50 \mathrm{~Gb} / \mathrm{s}$ and (b) corresponding RMSE of the output waveform and eye diagrams for sample $t_{\text {off }}$ values.

Overall, the results in Fig. 17(b) indicate that greatly improved tolerance to the timing offset $t_{\text {off }}$ can be achieved with the proposed adaptive gain scheme. Low RMSE values $\leq 0.05$ are obtained in the range -0.3 UI to +0.65 UI., which is roughly a 6 times improvement over the case when no gain adaptation is applied (Fig. 16).

\section{DISCUSSION}

A few points related to the application of the $\mathrm{ABCD}$ approximation and Stretched A methods are discussed below.

(i) Choice of response time $T_{c}$ : The choice of the desired response time $T_{c}$ affects the shape of the sub-current components required to remove the laser non-linearity. As a result, it has a direct impact on the bandwidth requirements for the driving electronics and electrical connections to the laser. Throughout this article, the proposed methods have been implemented for a target response time $T_{c}$ of $0.75 \times T$ as this provides open eye diagrams [Fig. 11(b)] without the need for a powerful linear equalizer. In practice however, larger values closer to $1.0 \times T$ might be preferred as this relaxes the bandwidth requirements for the current modulation waveforms that have to be generated. The proposed methods work equally well for such $T_{c}$ values, but the eye opening of the output optical waveform is smaller, requiring therefore a larger amount of linear equalisation (resulting in larger NEF) to fully open the eye diagrams. To illustrate this, the Stretched A method is applied on the modelled VCSEL for a desired $T_{c}$ of $1.0 \times T$ and a PRBS-7 input at the same data rate of $50 \mathrm{~Gb} / \mathrm{s}$. The eye diagram of the output optical waveform obtained for the ideal back-calculated current and via the Stretched A approximation method before and after the application of the 11-tap FFE are shown in Fig. 18. Clearly the method works equally well for the larger desired response time $T_{c}$. The linear FFE is able to equalize the output waveform and fully open the eye diagram but has a larger NEF due to the smaller inner eye height of the transmitted output waveform [Fig. 18(c)]. However, it should be recognized that if a receive decision feedback equalizer (DFE) were used then the NEF could be significantly reduced.
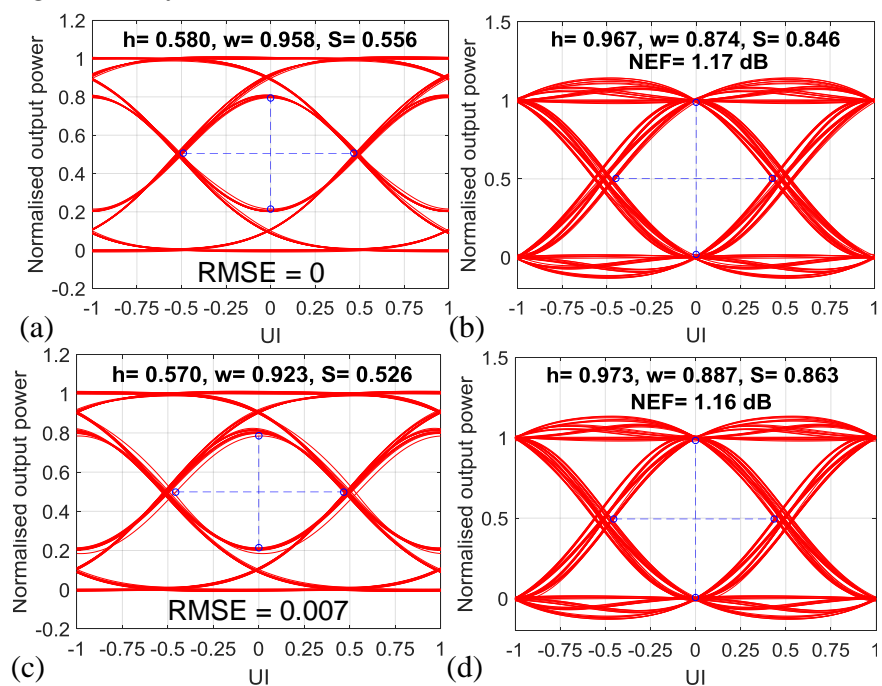

Fig. 18. Eye diagrams with metrics noted for the ABCD Stretched A method and a target response time $T_{c}$ of $1.0 \times T$ : (a-b) for the ideal back-calculated current $I_{b c}$ (c-d) when the approximate $I_{A}^{s t, o p t}$ and $\widetilde{I_{B}}$ are used before (a) and (c), and after (b) and (d) the application of the 11-tap FFE.

(ii) Pattern length: A short PRBS-7 has been used throughout this work to demonstrate the principle and benefits of the Stretched A method. The method works equally well for longer patterns as the generated modulating current removes the non-linearity for each transition via the ${\widetilde{I_{A}}}_{A} / I_{A}^{\text {st,opt }}$ and ${\widetilde{I_{B}}}$ components. To illustrate this point, the data transmission of a PRBS-15 is simulated at $50 \mathrm{~Gb} / \mathrm{s}$ using the same VCSEL and desired response time $T_{c}$ of $0.75 \times T$. The T-spaced 11-tap FFE is again applied to remove the linear distortion of the output optical waveform. Fig. 19 shows the eye diagram of the output optical waveform when no non-linear correction is applied (un-corrected waveform), and when the ideal back-calculated current and the approximate $\widetilde{I_{b c}}(t)$ obtained through the Stretched A method are applied to the VCSEL. The eye diagrams obtained clearly demonstrate that the proposed method works equally well for longer patterns. 

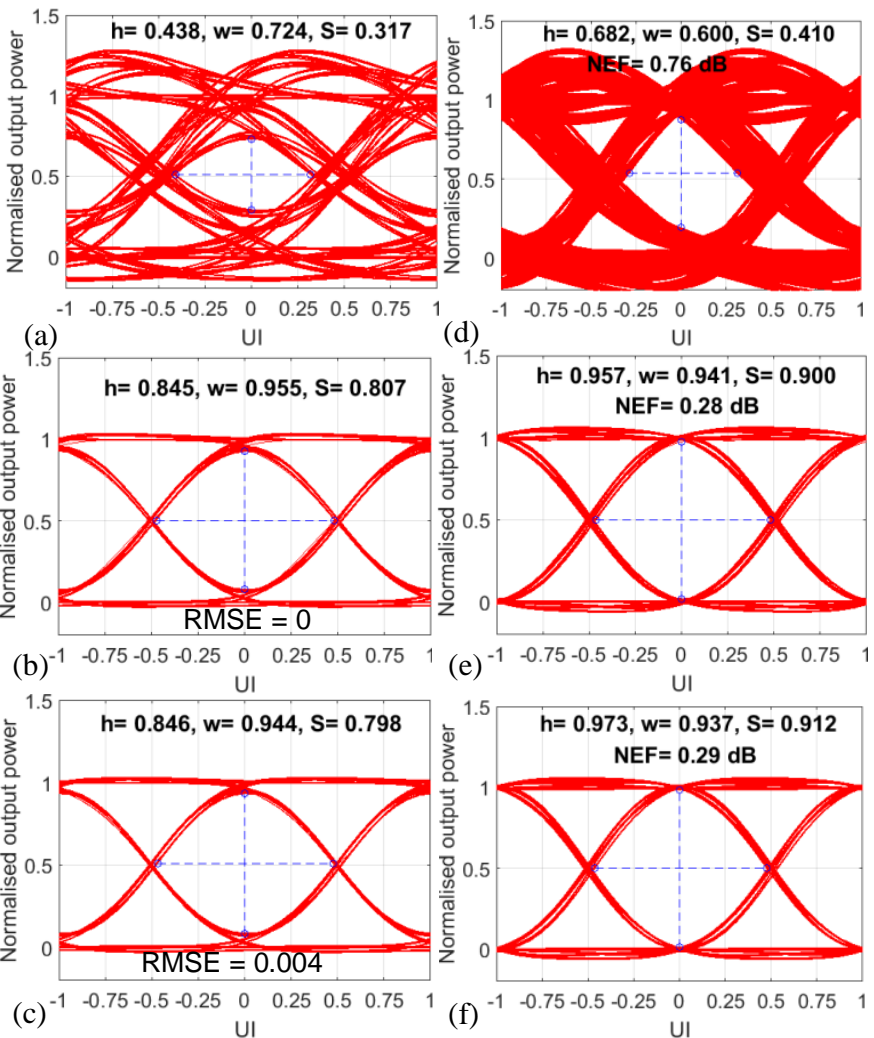

(d)

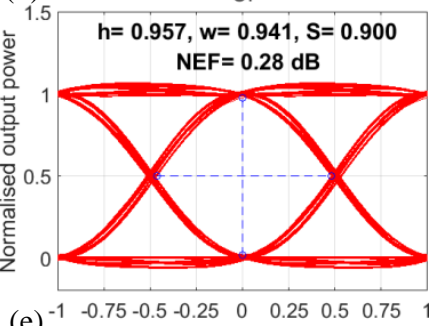

(e)

UI

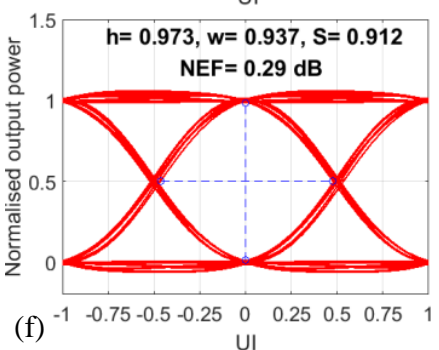

$\mathrm{UI}$

Fig. 19. Eye diagrams with metrics noted of the output optical waveform before (a-c) and after (d-f) the application of the 11-tap FFE for the data transmission of PRBS-15 at $50 \mathrm{~Gb} / \mathrm{s}$ when (a and d) no non-linear correction is applied, ( $b$ and e) for the ideal backcalculated current $I_{b c}$ and (c and f) for the approximate backcalculated current $\widetilde{I_{b c}}$ obtained via the Stretched A method.

(iii) $I_{A}^{s t}$ parameters: The optimum values of the $\gamma$ and $d t$ parameters of the approximate $I_{A}^{s t}$ component are obtained by finding the values that yield either the lowest RMSE value for the output optical waveform $P(t)$ when compared with the target waveform (Fig. 10) or the larger effective area $S$ for the eye diagram after the application of the FFE (Fig. 12). However, it is also possible to obtain a good estimate of the optimum values by comparing the $I_{A}^{s t}$ waveform with the ideal one $I_{A}$ using a RMSE metric. This method has the advantage that does not require the generation of optical waveforms to obtain a good estimate of the optimum values, but could provide slightly sub-optimal values. This is due to the fact that not all parts of the $I_{A}^{s t}$ waveform are equally important for the generation of the desired linear output waveform (e.g. the rising and falling edges appear to be more important than the timing of the zero crossing). This method has been carried out for data transmission at $50 \mathrm{~Gb} / \mathrm{s}$ using the desired response time $T_{c}$ of $0.75 \times \mathrm{T}$ and the same simulation parameters as those employed for section IV.A and the results are shown in Fig. 20. The purple dot in the plot indicates the point of minimum RMSE for the $I_{A}^{s t}$ component. This is achieved for $\gamma=$ $1.05, d t=0.095$ UI. The other two metrics presented in section IV.A yield $\gamma=1.07, d t=0.08 \mathrm{UI}$ for the optimum $I_{A}^{s t}$ parameters. This point is noted with a yellow dot in Fig. 20. It can be clearly noticed that the method based on the comparison of $I_{A}^{s t}$ and $I_{A}$ provides a very good estimate of the optimum parameter values. Given the large tolerances of the
Stretched A method (Fig. 10 and Fig. 12), similarly good performance is expected from the two sets of parameters in practice. This method can be very useful in practical implementations of the Stretched A method as near optimum parameters can be obtained for $I_{A}^{s t}$ using only the laser parameters (known either by design or measurements).

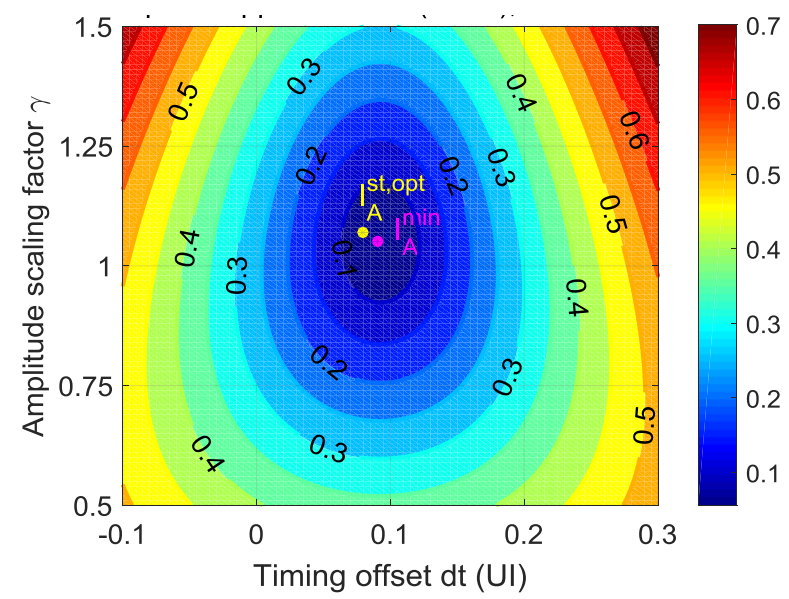

Fig. 20. RMSE $\left(\times 10^{-3}\right)$ of the $I_{A}^{s t}$ component when compared with the ideal sub-current $I_{A}$ as the function of the parameters $\gamma$ and $d t$. The purple dot indicates the point where the minimum RMSE is obtained.

(iv) By simulation, it can be shown that if a T-spaced FFE having 11 taps is used to pre-distort the NRZ modulation current of the VCSEL, the optical eye metrics $(h, w, S)$ at 50 $\mathrm{Gb} / \mathrm{s}$ are $(0.81,0.66,0.54)$. Therefore, the Stretched A method outperforms linear pre-distortion by the FFE.

\section{CONCLUSIONS}

A new linearization method for optical transmitters based on directly modulated lasers, named the "Stretched A" method is presented. The method approximates the ideal modulating current that produces a linear optical output waveform with a current waveform that provides sufficiently good correction of the laser non-linearity. The theory and the derivation of the set of equations of the proposed method are reported. Simulation studies focussed on VCSEL-based optical transmitters and NRZ-based $50 \mathrm{~Gb} / \mathrm{s}$ data transmission are presented. The results demonstrate that this new method produces an optical waveform which is very close to the ideal linear output waveform with little residual non-linearity and with large enough tolerances to make this scheme viable in real-world systems.

\section{REFERENCES}

[1] Cisco, "Cisco Global Cloud Index: Forecast and Methodology, 20162021 White Paper," 2018.

[2] G. Belfiore, M. Khafaji, R. Henker, and F. Ellinger, "A 50 Gb/s $190 \mathrm{~mW}$ Asymmetric 3-Tap FFE VCSEL Driver," IEEE Journal of Solid-State Circuits, vol. 52, pp. 2422-2429, 2017.

[3] K. Ohhata, H. Imamura, Y. Takeshita, K. Yamashita, H. Kanai, and N. Chujo, "Design of a $4 \times 10 \mathrm{~Gb} / \mathrm{s}$ VCSEL Driver Using Asymmetric Emphasis Technique in 90-nm CMOS for Optical Interconnection," IEEE Transactions on Microwave Theory and Techniques, vol. 58, pp. 1107$1115,2010$.

[4] A. S. Ramani, S. Nayak, and S. Shekhar, "A Differential Push-Pull Voltage Mode VCSEL Driver in 65-nm CMOS," IEEE Transactions on Circuits and Systems I: Regular Papers, vol. 66, pp. 4147-4157, 2019. 
[5] M. Raj, M. Monge, and A. Emami, "A Modelling and Nonlinear Equalization Technique for a $20 \mathrm{~Gb} / \mathrm{s} 0.77 \mathrm{pJ} / \mathrm{b}$ VCSEL Transmitter in $32 \mathrm{~nm}$ SOI CMOS," IEEE Journal of Solid-State Circuits, vol. 51, pp. 1734-1743, 2016.

[6] D. Liang, A. Roshan-Zamir, Y.-H. Fan, C. Zhang, B. Wang, A. Descos, W. Shen, K. Yu, C. Li, G. Fan, G. Kurczveil, Y. Hu, Z. Huang, M. Fiorentino, S. Kumar, S. M. Palermo, and R. G. Beausoleil, "FullyIntegrated Heterogeneous DML Transmitters for High-Performance Computing," Journal of Lightwave Technology, vol. 38, pp. 3322-3337, 2020.

[7] U. Hecht, N. Ledentsov, P. Scholz, M. Agustin, P. Schulz, N. N. Ledentsov, and F. Gerfers, "Non-Linear PAM-4 VCSEL Equalization and $22 \mathrm{~nm}$ SOI CMOS DAC for $112 \mathrm{Gbit} / \mathrm{s}$ Data Transmission," in 12th German Microwave Conference (GeMiC), 2019, pp. 115-118.

[8] J. M. Castro, R. J. Pimpinella, B. Kose, Y. Huang, A. Novick, and B. Lane, "Eye skew modeling, measurements and mitigation methods for VCSEL PAM-4 channels at data rates over $66 \mathrm{~Gb} / \mathrm{s}$," in Optical Fiber Communications Conference and Exhibition (OFC), 2017, pp. 1-3.

[9] Y. Yu, M. R. Choi, T. Bo, Z. He, Y. Che, and H. Kim, "Low-Complexity Second-Order Volterra Equalizer for DML-Based IM/DD Transmission System," Journal of Lightwave Technology, vol. 38, pp. 1735-1746, 2020.

[10] J. Liu, K. Chi, C. Wei, T. Lin, C. Chuang, X. Chen, J. Shi, and J. Chen, "High bit-rate distance product of $128 \mathrm{Gbps.km}$ 4-PAM transmission over 2-km OM4 fiber using an 850-nm VCSEL and a Volterra nonlinear equalizer," in Optical Fiber Communications Conference and Exhibition (OFC), 2017, pp. 1-3.

[11]N. Stojanovic, F. Karinou, Z. Qiang, and C. Prodaniuc, "Volterra and Wiener Equalizers for Short-Reach 100G PAM-4 Applications," Journal of Lightwave Technology, vol. 35, pp. 4583-4594, 2017.

[12]L. Ge, W. Zhang, C. Liang, and Z. He, "Threshold-Based Pruned Retraining Volterra Equalization for $100 \mathrm{Gbps/Lane} \mathrm{and} \mathrm{100-m} \mathrm{Optical}$ Interconnects Based on VCSEL and MMF," Journal of Lightwave Technology, vol. 37, pp. 3222-3228, 2019.

[13]A. Tyagi, T. Iwai, K. Yu, B. Wang, W. Sorin, S. Mathai, M. Tan, and S. Palermo, "A $50 \mathrm{~Gb} / \mathrm{s}$ PAM-4 VCSEL Transmitter With 2.5-Tap Nonlinear Equalization in 65-nm CMOS," IEEE Photonics Technology Letters, vol. 30, pp. 1246-1249, 2018.

[14]P. Gou, L. Zhao, K. Wang, W. Zhou, and J. Yu, "Nonlinear Look-Up Table Predistortion and Chromatic Dispersion Precompensation for IM/DD PAM-4 Transmission," IEEE Photonics Journal, vol. 9, pp. 1-7, 2017.

[15]A. S. Karar, M. Ya ̃̃ \pm ez, Y. Jiang, J. C. Cartledge, J. Harley, and K. Roberts, "Electronic dispersion pre-compensation for $10.709-\mathrm{Gb} / \mathrm{s}$ using a look-up table and a directly modulated laser," Optics Express, vol. 19, pp. B81-B89, 2011.

[16] A. S. Karar, J. C. Cartledge, J. Harley, and K. Roberts, "Electronic PreCompensation for a 10.7-Gb/s System Employing a Directly Modulated Laser," Journal of Lightwave Technology, vol. 29, pp. 2069-2076, 2011.

[17]S. Warm, C. Bunge, T. Wuth, and K. Petermann, "Electronic Dispersion Precompensation With a 10-Gb/s Directly Modulated Laser," IEEE Photonics Technology Letters, vol. 21, pp. 1090-1092, 2009.

[18]K. Burse, R. N. Yadav, and S. C. Shrivastava, "Channel Equalization Using Neural Networks: A Review," IEEE Transactions on Systems, Man, and Cybernetics, Part C (Applications and Reviews), vol. 40, pp. 352-357, 2010.

[19]J. Estaran, R. Rios-Mueller, M. A. Mestre, F. Jorge, H. Mardoyan, A. Konczykowska, J. Dupuy, and S. Bigo, "Artificial Neural Networks for Linear and Non-Linear Impairment Mitigation in High-Baudrate IM/DD Systems," in 42nd European Conference on Optical Communication (ECOC), 2016, pp. 1-3.

[20]T. A. Eriksson, H. Bulow, and A. Leven, "Applying Neural Networks in Optical Communication Systems: Possible Pitfalls," IEEE Photonics Technology Letters, vol. 29, pp. 2091-2094, 2017.

[21]L. Illing and M. B. Kennel, "Shaping current waveforms for direct modulation of semiconductor lasers," IEEE Journal of Quantum Electronics, vol. 40, pp. 445-452, 2004.

[22]T. Yazaki, N. Chujo, H. Yamashita, T. Takemoto, Y. Lee, and Y. Matsuoka, "25-Gbps x 4 optical transmitter with adjustable asymmetric pre-emphasis in 65-nm CMOS," in IEEE International Symposium on Circuits and Systems (ISCAS), 2014, pp. 2692-2695.

[23] V. Kozlov and A. C. Carusone, "A 15Gb/s AC-Coupled VCSEL Driver with Waveform Shaping in 65nm CMOS," in IEEE Compound Semiconductor Integrated Circuit Symposium (CSICS), 2015, pp. 1-4.
[24]L. A. Valenzuela, H. Andrade, N. Hosseinzadeh, A. Maharry, C. L. Schow, and J. F. Buckwalter, "A $2.85 \mathrm{pJ} / \mathrm{bit}, 52-\mathrm{Gbps}$ NRZ VCSEL Driver with Two-Tap Feedforward Equalization," in IEEE/MTT-S International Microwave Symposium (IMS), 2020, pp. 209-212.

[25]A. S. Karar, "Electronic bandwidth enhancement of directly modulated semiconductor lasers," Optics Communications, vol. 439, pp. 137-140, 2019.

[26]G. M. Choudhury, J. Robert Lingle, J. Kamino, R. Shubochkin, D. Vaidya, D. Braganza, and Y. Sun, "Standards, Technology, Market and Industry Trends and Future Direction for MMF," in IWCS 2019 International Cable \& Connectivity Symposium. vol. paper 5.1, 2019, pp. $1-12$.

[27]J. A. Tatum, D. Gazula, L. A. Graham, J. K. Guenter, R. H. Johnson, J. King, C. Kocot, G. D. Landry, I. Lyubomirsky, A. N. MacInnes, E. M. Shaw, K. Balemarthy, R. Shubochkin, D. Vaidya, M. Yan, and F. Tang, "VCSEL-Based Interconnects for Current and Future Data Centers," Journal of Lightwave Technology, vol. 33, pp. 727-732, 2015.

[28]D. G. Cunningham and I. H. White, "Advances in local area optical data communication systems," Reports on Progress in Physics, vol. 83, p. 075101, 2020.

[29] N. Eiselt, H. Griesser, J. Wei, R. Hohenleitner, A. Dochhan, M. Ortsiefer, M. H. Eiselt, C. Neumeyr, J. J. V. Olmos, and I. T. Monroy, "Experimental Demonstration of $84 \mathrm{~Gb} / \mathrm{s}$ PAM-4 Over up to $1.6 \mathrm{~km}$ SSMF Using a 20-GHz VCSEL at $1525 \mathrm{~nm}$," Journal of Lightwave Technology, vol. 35, pp. 1342-1349, 2017.

[30]N. Ledentsov, Å. Chorchos, V. A. Shchukin, V. P. Kalosha, J. P. Turkiewicz, and N. N. Ledentsov, "Development of VCSELs and VCSEL-based Links for Data Communication beyond $50 \mathrm{~Gb} / \mathrm{s}$," in Optical Fiber Communication Conference (OFC) 2020, San Diego, California, 2020, p. M2A.3.

[31]M. J. Li, K. Li, X. Chen, S. K. Mishra, A. A. Juarez, J. E. Hurley, J. S. Stone, C. H. Wang, H. T. Cheng, C. H. Wu, H. C. Kuo, C. T. Tsai, and G. R. Lin, "Single-Mode VCSEL Transmission for Short Reach Communications," Journal of Lightwave Technology, vol. 39, pp. 868880, 2021.

[32]IEEE 802.3 Ethernet Working Group, 400 Gigabit Ethernet Call-forInterest Consensus, IEEE 802, 2013.

[33]J. Ingham and R. Murty, "Towards technical feasibility of $100 \mathrm{~Gb} / \mathrm{s}$ per lane optical PMDs supporting 100 m OM4 MMF," IEEE P802.3db 100 $\mathrm{Gb} / \mathrm{s}, 200 \mathrm{~Gb} / \mathrm{s}$, and $400 \mathrm{~Gb} / \mathrm{s}$ Short Reach Fiber Task Force Ad Hoc Teleconference, 2020,
https://www.ieee802.org/3/db/public/adhoc/presentations/ingham_3db_ad hoc_01a_062520.pdf.

[34] A. S. Karar, Y. Jiang, J. C. Cartledge, J. Harley, D. J. Krause, and K. Roberts, "Electronic precompensation of the nonlinear distortion in a 10 $\mathrm{Gb} / \mathrm{s}$ 4-ary ASK directly modulated laser," in 36th European Conference and Exhibition on Optical Communication (ECOC), 2010, pp. 1-3.

[35]G. D. Brown, "Bandwidth and rise time calculations for digital multimode fiber-optic data links," Journal of Lightwave Technology, vol. 10, pp. 672-678, 1992.

[36] M. C. Nowell, D. G. Cunningham, D. C. Hanson, and L. G. Kazovsky, "Evaluation of $\mathrm{Gb} / \mathrm{s}$ laser based fibre LAN links: Review of the Gigabit Ethernet model," Optical and Quantum Electronics, vol. 32, pp. 169-192, 2000.

[37]D. G. Cunningham and P. Dawe, "Review of the 10Gigabit Ethernet Link Model," Avago Technologies White Paper, vol. paper AV02-2485EN, pp. $1-10,2002$.

[38] "IEEE 802.3ae 10G Ethernet optical link budget spreadsheet," available online:

https://www.ieee802.org/3/ae/public/adhoc/serial_pmd/documents/10GE PBud3 1_16a.xls

[39] J. Petrilla, Example MMF link model "ExampleMMF LinkModel 130503.xlsx" (2013), available http://www.ieee802.org/3/bm/public/may13/index.html

[40]P. Westbergh, J. S. Gustavsson, A. Haglund, M. Skold, A. Joel, and A. Larsson, "High-Speed, Low-Current-Density $850 \mathrm{~nm}$ VCSELs," IEEE Journal of Selected Topics in Quantum Electronics, vol. 15, pp. 694-703, 2009.

[41]A. Mutig and D. Bimberg, "Progress on High-Speed 980-nm VCSELs for Short-Reach Optical Interconnects," Advances in Optical Technologies, vol. 2011, p. 290508, 2011.

[42]P. Pepeljugoski, S. E. Golowich, A. J. Ritger, P. Kolesar, and A. Risteski, "Modeling and simulation of next-generation multimode fiber links," Journal of Lightwave Technology, vol. 21, pp. 1242-1255, 2003. 